\title{
Islam hétérodoxe et christianisme en Grèce
}

Tabous, identités religieuses et discours nationaux

Heterodox Islam and Christianism in Greece: Taboos, Religious Identities and

National Discourse

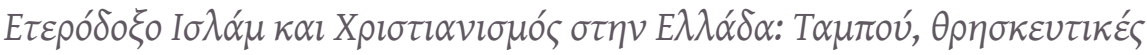

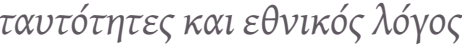

\section{Isabelle Dépret}

\section{OpenEdition}

Journals

Édition électronique

URL : https://journals.openedition.org/ceb/8887

DOI : $10.4000 / c e b .8887$

ISSN : 2261-4184

\section{Éditeur}

INALCO

Édition imprimée

ISBN : 978-2-85837-229-0

ISSN : 0290-7402

\section{Référence électronique}

Isabelle Dépret, «Islam hétérodoxe et christianisme en Grèce », Cahiers balkaniques [En ligne], 43 |

2015, mis en ligne le 26 juillet 2017, consulté le 06 juillet 2021. URL : http://journals.openedition.org/ ceb/8887 ; DOI : https://doi.org/10.4000/ceb.8887

Ce document a été généré automatiquement le 6 juillet 2021.

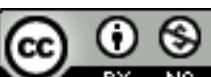

Cahiers balkaniques est mis à disposition selon les termes de la Licence Creative Commons Attribution - Pas d'Utilisation Commerciale 4.0 International. 


\title{
Islam hétérodoxe et christianisme en Grèce
}

\author{
Tabous, identités religieuses et discours nationaux \\ Heterodox Islam and Christianism in Greece: Taboos, Religious Identities and \\ National Discourse

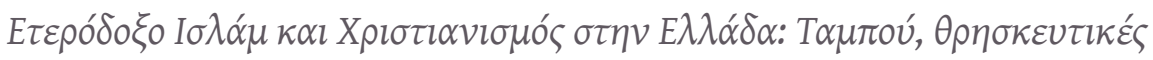 \\ $\tau \alpha v \tau o ́ \tau \eta \tau \varepsilon \varsigma \kappa \alpha l \varepsilon \theta v i k o ́ \varsigma \lambda o ́ \gamma o \varsigma$
}

Isabelle Dépret

\section{Introduction}

1 En mai 2011, un membre de la communauté bektachie du village de Roussa/Ruşanlar, en Thrace grecque, confiait :

Je suis bektachi, mais, dans un environnement majoritairement sunnite, nous avons parfois caché nos spécificités. Une partie des bektachis sont devenus sunnites (...) Nous avons chez nous des mariages avec des sunnites. En général, ces mariages sont un échec. (...) Je suis Turc, mais mes grands-parents parlaient le bulgare (...). Les autorités grecques disent que je suis Pomaque (...). Je n'accepte pas que l'on décide à ma place quelle est mon identité. Même si je suis Turc, lorsque je suis face aux services de douanes grecques ou bien si je veux obtenir un permis auprès de l'administration hellénique, je déclare que je suis Pomaque. Si je leur disais que je suis Turc, je n'aurais que des soucis ${ }^{1}$.

2 Ces remarques d'un citoyen grec musulman «hétérodoxe " suggèrent les enjeux associés aux groupes minoritaires dans une région longtemps écrasée par les nationalismes. Ces propos, d'une grande lucidité, soulignent la complexité des constructions identitaires, leur fluidité, leurs possibles réajustements en fonction de contraintes ou d'opportunités sociales. J'explorerai ces deux questions en me penchant sur deux communautés minoritaires en Grèce: les alévis d'une part, les Pomaques de l'autre. 
Dans un État où l'orthodoxie se pose comme religion dominante, l'alévisme constitue un petit groupe au sein de la «minorité musulmane» de Thrace ${ }^{2}$. Ce courant religieux et culturel s'inscrit dans un espace transfrontalier qui plonge ses racines dans l'histoire ottomane de la région. Quant aux musulmans de langue maternelle slave/bulgare - les Pomaques - ils participent eux aussi de l'islam balkanique: présents surtout en Bulgarie, en Turquie, ils seraient environ 30000 au nord-est de la Grèce à la fin du $\mathrm{xx}^{\mathrm{e}}$ siècle $^{3}$.

4 Je m'attacherai, tout d'abord, à préciser les contours de ces deux groupes musulmans de Grèce, deux groupes parfois articulés. J'insisterai sur leur situation d'incertitude identitaire et leur tiraillement aujourd'hui entre plusieurs tendances contraires. Je me centrerai ensuite sur un litige survenu, au début $d u x x^{\mathrm{e}}$ siècle, en espace local et scolaire. Je tenterai de mieux saisir le poids symbolique de ce litige en l'inscrivant dans un cadre intellectuel, politique, en le reliant à des logiques d'acculturation, dans une région pluriculturelle en mutation.

\section{L'alévisme-bektachisme en Thrace grecque, islam hétérodoxe et carrefour identitaire}

\section{Un autre islam longtemps ignoré en Grèce}

\section{Constructions nationales dans les Balkans : le paramètre confessionnel}

L'étude des pays d'Europe du Sud-Est au Xxe siècle ne peut négliger l'héritage ottoman ${ }^{4}$. À partir du XIX $x^{e}$ siècle, la formation d'États-nations sur l'ancien territoire des Sultans a accéléré un processus de sécularisation et recomposé les liens entre religion, identité, pouvoirs et communautés ${ }^{5}$. Si dans l'Empire, la confession jouait un rôle social structurant, par la suite, l'appartenance religieuse a continué à peser, parfois de manière décisive, dans la consolidation des idéologies nationales ${ }^{6}$.

6 Aux $\mathrm{XIX}^{\mathrm{e}}$ et $\mathrm{XX}^{\mathrm{e}}$ siècles, l'école, l'armée, les médias ou encore les commémorations célébrées à l'échelle locale ont souvent servi des projets politiques, celui, notamment, de produire, d'exalter un patriotisme propre à légitimer l'obéissance « volontaire » des citoyens à l'État-nation et à ses lois ${ }^{7}$. Si l'emprise du religieux dans la vie sociale a décliné au $\mathrm{Xx}^{\mathrm{e}}$ siècle, l'association entre une nation et une confession particulière a parfois vulnérabilisé les porteurs d' "altérité", face aux autorités confessionnelles majoritaires ou encore face au politique ${ }^{8}$. Ceci a notamment valu en Grèce.

\section{L'islam en Grèce : la religion de l'autre?}

7 Aux XIX et $x^{e}$ siècles, la formation de l'État grec s'est, en effet, en partie structurée autour du lien hellénisme-christianisme orthodoxe ${ }^{9}$. À ce lien, fondement d'un imaginaire national, a correspondu une certaine confusion entre les notions "d'Ottoman ", de "musulman », de " Turc », trois termes rejetés, certes abusivement, dans « l'étrangeté » absolue ${ }^{10}$. Jusqu'au début du $\mathrm{xx}^{\mathrm{e}}$ siècle, le terme de Turc véhicule un sens principalement confessionnel ${ }^{11}$.

Pourtant, l'islam a toujours persisté en Grèce : si après 1947, il ne concerne qu'une petite minorité des citoyens ( 1 à $2 \%$ de la population totale), après l'annexion des 
« Nouvelles Provinces » - Macédoine, Épire, Crète, Thrace occidentale - il représentait 700000 personnes, soit $14 \%$ de la population du pays ${ }^{12}$.

9 Ces rapports particuliers à l'islam ${ }^{13}$ transparaissent dans les archives de l'État - c'est-àdire dans le regard de l'administration hellénique au $\mathrm{Xx}^{\mathrm{e}}$ siècle $^{14}$. Au-delà des principes libéraux souvent privilégiés par la législation, la presse ou encore les rapports d'acteurs étatiques, véhiculent de manière récurrence l'idée selon laquelle les musulmans du pays ne peuvent représenter de « vrais » Grecs et $a$ fortiori de sûrs patriotes ${ }^{15}$.

Ces regards méfiants, dépréciatifs ont valu en particulier pour les musulmans de Thrace occidentale. Ce groupe - environ 120000 personnes - a été exempté des échanges démographiques gréco-turcs de 1923, à l'instar des chrétiens orthodoxes Rums d'Istanbul, des îles d'Imvros (Gökçeada tr.), de Ténédos (Bozcaada tr.), en République turque ${ }^{16}$. Face à d'autres éléments culturels, c'est le facteur confessionnel qui a donc prévalu comme critère de ces déplacements massifs forcés. Les populations non «échangées »-Rums d'Istanbul et musulmans de Thrace grecque - ont été protégées en droit international en tant, désormais, que minorités ${ }^{17}$.

11 Si la Thrace grecque a été repeuplée par des immigrés chrétiens dès l'entre-deuxguerres $^{18}$, les musulmans - largement majoritaires dans cette région au début du $\mathrm{xx}^{\mathrm{e}}$ siècle $^{19}$ - résident surtout dans deux des trois départements de la région : ceux de Xanthi et du Rhodope. En 1951 - lors du dernier recensement présentant des données religieuses - les musulmans sont fort présents dans ces deux départements : près de $45 \%$ de la population totale dans le nome de Xanthi, près de $56 \%$ dans celui de Rhodope $^{20}$. Selon Byron Kotzamanis, la répartition musulmans-chrétiens aurait peu évolué au cours des 50 années qui suivent ${ }^{21}$, en dépit d'un exode rural et de l'afflux de chrétiens ("russes ») pontiques dans les années 1990²2.

$12 \mathrm{Au} \mathrm{Xx}$ siècle, les musulmans de Thrace ont formé une population principalement rurale, moins scolarisée en moyenne que la population chrétienne, restée « traditionnelle » dans ses modes de fonctionnement ${ }^{23}$. Une évolution nette est en cours depuis les années $1990^{24}$. Outre les chrétiens orthodoxes, aujourd'hui les plus nombreux, la région compte une très petite communauté arménienne et quelques juifs ${ }^{25}$. Jadis dynamique, la communauté juive de la région a, en effet, été quasi intégralement déportée en 1943, durant l'administration bulgaro-nazie ${ }^{26}$.

13 Ainsi, la Thrace présente-t-elle un visage particulier de la Grèce $a u x^{e}$ siècle. Dans un pays où les monuments ottomans ont d'abord fait l'objet de destructions ou de réemplois aux $\mathrm{XIX}^{\mathrm{e}}$ et $\mathrm{XX}^{\mathrm{e}}$ siècles ${ }^{27}$, cette région frontalière ${ }^{28}$ compte en effet plus de 300 mosquées, plus de 350 imams ainsi que trois muftis officiels - experts en droit coranique - qui, rémunérés par l'État, ont conservé des compétences judiciaires en droit familial ${ }^{29}$. La région admet également un système éducatif primaire et secondaire réservé à la "minorité ", où les cours sont dispensés en grec et en turc. L'éducation religieuse est ici centrée sur l'islam sunnite ${ }^{30}$.

14 Si depuis les années 1990, l'administration grecque insiste sur l'hétérogénéité ethnique des musulmans de Thrace - personnes « d'origine turque », Pomaques et Tsiganes ${ }^{31}$ - il existe d'autres facteurs de différenciation au sein de la minorité: les musulmans sunnites - écrasante majorité - coexistent avec de petites communautés bektachies/ alévies. 


\section{Islam et alévisme en Thrace}

Religion et culture "syncrétiques » selon l'historienne Irène Mélikoff ${ }^{32}$, l'alévisme s'inscrit dans la mouvance de l'islam soufi, mystique ${ }^{33}$. Il associe des éléments issus du chiisme à d'autres influences, dont le chamanisme et, pour certains, le christianisme ${ }^{34}$.

En Anatolie et en Thrace, l'alévisme est souvent associé au bektachisme, cet ordre religieux jadis ésotérique, influent dans l'Empire ottoman avant $1826^{35}$. Les deux courants se rattachent en effet au saint Haci Bektach Veli, figure du XIII ${ }^{e}$ siècle et acteur d'un long processus d'islamisation des Balkans ${ }^{36}$. Alévisme et bektachisme possèdent nombre d'éléments culturels communs. Pour Mélikoff, l'alévisme représenterait une sorte de version populaire du bektachisme ${ }^{37}$.

Les liens de l'alévisme au chiisme transparaissent dans la forte valorisation de la figure d'Ali, gendre et cousin du prophète Mahomet; dans la vénération des Douze Imams. De ses liens au soufisme témoignent la conviction que le texte coranique, comme toute réalité admettrait à la fois un sens «extérieur " (zahir) et «intérieur/caché » (batin). L'accession à un état spirituel permettant d'accéder à cette connaissance cachée est donc recherchée ${ }^{38}$. Des liens au soufisme témoignent cette logique mystique, initiatique, l'importance de la musique et de la danse rituelle lors des cérémonies religieuses $(\mathrm{cem})^{39}$.

\section{Une tradition ancienne étiolée depuis le $\mathrm{XIX}$ siècle}

\section{Une tradition spécifique en Thrace}

Si un document évaluait l'islam hétérodoxe à près de $10 \%$ de la population musulmane en Thrace ${ }^{40}$, les alévis ne représentent stricto sensu qu'environ 3000 personnes au début $\mathrm{du} \mathrm{xxI}^{\mathrm{e}}$ siècle $^{41}$ : celles-ci résident principalement dans une série de villages montagneux situés au nord du département de l'Évros et, dans une moindre mesure, de Xanthi et du Rhodope. Auto-désignés comme alévis-bektachis, ils sont aussi appelés Kizilbash (tête rouge) dans le nord de Xanthi, en référence à ces anciens militants chiites au turban rouge, liés à la fondation de la dynastie safavide iranienne ${ }^{42}$.

Le bektachisme était très présent en Thrace à l'époque ottomane - spécialement dans la région d'Alexandroúpolis, sur le littoral, mais aussi dans les montagnes du Rhodope ${ }^{43}$. L'installation, à partir $\mathrm{du} \mathrm{Xv} \mathrm{v}^{\mathrm{e}}$ siècle, de derviches s'est aussi inscrite dans une logique de consolidation des terres conquises par les Sultans, des terres alors largement chrétiennes. Le développement du bektachisme est dès lors marquant en Thrace, autorisant des croyances et rites moins stricts que ceux définis par l'islam sunnite officiel $^{44}$.

Du XIV e siècle au XIX siècle, dans les Balkans, certains tekkes - foyers spirituels, lieux d'accueil de voyageurs, espaces agricoles parfois très prospères - participent de l'économie agraire, des activités sociales des fondations pieuses (wakfs), rouages de la société ottomane ${ }^{45}$. Après la dissolution de l'ordre des Janissaires, en 1826, le déclin des tekke bektachis est amorcé au $\mathrm{XIX}^{\mathrm{e}}$ siècle ${ }^{46}$. Le courant s'est surtout étiolé au $\mathrm{Xx}^{\mathrm{e}}$ siècle, perdant ses fidèles, ses ressources économiques, ses réseaux anciens. Les réformes agraires menées au cours des années 1920-1950 en Grèce, des confiscations de biens non exploités ont peu à peu privé les tekke bektachis ${ }^{47}$ de l'essentiel de leurs propriétés foncières ${ }^{48}$. Selon l'historien Evstratios Zegginis, dans les années 1980, la Thrace 
compterait plusieurs dizaines de tekke, certains à l'état de vestige ou largement laissés à l'abandon ${ }^{49}$. Le poids du sunnisme s'est d'autant renforcé, soutenu par le mufti, interlocuteur légal de l'État grec ${ }^{50}$.

\section{Entre fermeture et réseaux transfrontaliers}

Des entretiens réalisés, en 2010 et 2011, auprès d'alévis de l'Évros signalent à quel point la mémoire de la persécution reste présente - ou du moins affirmée : mémoire de la répression politique depuis les sultans ottomans ; méfiance à l'égard de l'État grec, qui valoriserait le christianisme; méfiance à l'égard du sunnisme, qui aurait falsifié le Coran et exercerait une domination injustifiée ${ }^{51}$. Les personnes interrogées se définissent comme musulmanes, revendiquant même leur appartenance à la tradition la plus authentique de l'islam ${ }^{52}$.

Compte tenu de cette culture de la suspicion, une partie des alévis tend d'abord à se présenter comme sunnite ${ }^{53}$. Dans les années 1980, le mufti de Xanthi condamne des pratiques jugées non orthodoxes : par exemple, la tradition de rendre visite à des saints locaux $\mathrm{x}^{54}$; celle de nouer des bouts de tissus et de les accrocher aux arbres entourant un lieu sacré; de jeter des pièces de monnaie ou de se presser le visage contre la tombe d'un saint, en invoquant son aide ${ }^{55}$. Ce rappel à l'ordre signale le maintien de ces pratiques dans l'islam populaire, ceci au-delà du groupe alévi stricto sensu ${ }^{56}$.

Les alévis-bektachis de Thrace s'inscrivent au carrefour des cultures turque-bulgaregrecque, avec des implications politiques. Une partie des musulmans de langue maternelle slave - les Pomaques - est alévie. D'autres alévis sont d'origine turque. Face aux « ennemis » du Nord puis de l'Est, Bulgarie et Turquie - l'espace septentrional de la Thrace a été classé sous supervision militaire de 1936 à $1995^{57}$. Ces facteurs ont longtemps placé les musulmans de cette région dans une double situation d'isolement et de relative extériorité au regard de l'État grec ${ }^{58}$. La circulation des personnes de cette zone était limitée, contrôlée par l'armée, la police et des bureaux culturels aux visées politiques ${ }^{59}$. Non protégées, ni par l'État ni par les autorités musulmanes légales, les identités alévies en Grèce semblent se percevoir comme menacées, tiraillées entre fermeture communautaire, assimilation au sunnisme, sécularisation et reconstitution de solidarités internationales ${ }^{60}$.

Ces phénomènes contradictoires peuvent notamment être observés autour de deux tekke associés : celui de Seyyit Ali Sultan, près du village de Roussa/Ruşanlar; celui d'Aşağı à Mikro Dereio/Küçük Derbent, dans l'Évros, non loin de la frontière grécobulgare. Du 6 mai - jour de la fête d'Ederlez, dédiée au prophète Hidir ${ }^{61}$ - à novembre, une série de fêtes religieuses, profanes et agraires ponctuent le calendrier des musulmans alévis ${ }^{62}$. Depuis les années 2000, certaines des fêtes les plus médiatisées occasions de repas (kurban) ou de petites foires - sont fréquentées par des personnes venues de Turquie, de Bulgarie ou d'autres régions balkaniques ${ }^{63}$. Cette fréquentation transfrontalière vaut, plus récemment, pour la fête du 8 novembre, dédiée au fils du saint bektachi local, au tekke de Roussa.

Le tekke de Roussa aurait été fondé en 1402 à la mémoire de Seyyid Ali Sultan surnommé Kızll Deli, le «Fou rouge » - un saint et guerrier des XIV ${ }^{\mathrm{e}}-\mathrm{XV}^{\mathrm{e}}$ siècles ${ }^{64}$. Lieu de culte important à l'époque ottomane, le tekke a représenté un vakıf riche au plan foncier jusqu'au $\mathrm{XIX}^{\mathrm{e}}$ siècle ${ }^{65}$. Dévasté au début $\mathrm{du} \mathrm{Xx}^{\mathrm{e}}$ siècle par les conflits bulgarogrecs, puis pendant la Seconde Guerre mondiale, privé par la suite de ses domaines, le 
dergâh de Seyyid Ali Sultan ne dispose que de quelques mètres carrés de terrain au début du xxI ${ }^{\mathrm{e}}$ siècle ${ }^{66}$. Il est aujourd'hui géré en tant que wakf ${ }^{67}$ par un comité local de musulmans alévis ${ }^{68}$. Il inclut une salle de prière; le cénotaphe (türbe) du saint, un réfectoire où apparaissent des portraits d'Ali ; une cuisine ; un espace d'abattage des animaux; une étable; l'ancienne résidence d'un baba, le dirigeant spirituel du lieu, devenue maison du gardien du mausolée ; un cimetière ${ }^{69}$. L'espace abrite également une petite mosquée, reflet d'une ouverture aux sunnites locaux ou de pratiques syncrétiques. Les alévis de Roussa/Ruşanlar - l'essentiel de la communauté villageoise - se définissent comme «Turcs », certains comme « Pomaques» et citoyens grecs. Aujourd'hui, la plupart parlent le turc ${ }^{70}$.

À la fin du $\mathrm{xx}^{\mathrm{e}}$ siècle, des associations turques, des fonctionnaires de l'État turc marquent de leur présence certaines fêtes alévies ${ }^{71}$. La " propagande turque » est alors déplorée parmi les Grecs chrétiens : les autorités turques ne chercheraient-elles pas à récupérer l'alévisme en dépouillant ces fêtes traditionnelles de leur caractère culturel ou religieux originels ${ }^{72}$ ? Par réaction, des représentants de l'État grec, des personnalités politiques, des associations, des acteurs du mouvement identitaire pomaque tiennent, eux aussi, à ré-occuper le terrain, longtemps délaissé, de ces festivités religieuses ou séculières, contribuant par là même à revaloriser cet héritage musulman hétérodoxe, perçu comme inoffensif, positif sinon utile du point de vue des « intérêts nationaux »"

Le double statut de membre de la minorité, d'hétérodoxe au regard du sunnisme dominant; un environnement rural, écarté, montagneux, une mémoire des conflits politiques, du rejet, ces facteurs éclairent des mécanismes de protection, de repli, la difficulté de se confier d'emblée à un étranger ${ }^{74}$. Parmi les interlocuteurs se reconnaissant, en 2010 et 2011, comme Bektachis-Alévis une partie -majoritaire présente cette identité comme religieuse ${ }^{75}$. D'autres, surtout urbains, posent cette appartenance comme culturelle: comme une tradition, une manière de penser, de vivre. Pour plusieurs hommes des villages de Roussa, des villes de Komotini, de Xanthi, le bektachisme inclurait une culture de la résistance aux pouvoirs établis, une ouverture, une tolérance religieuse et intellectuelle « intrinsèques ", l'éloignant de tout « fanatisme $»^{76}$. Pour d'autres enfin, l'identité bektachie-alévie est étroitement articulée à une appartenance ethno-nationale, turque ou slave ${ }^{77}$. Dans les villages, la plupart des femmes alévies sont voilées et s'insèrent dans un modèle familial patriarcal, certes en voie d'effritement ${ }^{78}$.

28 L'alévisme-bektachisme de Thrace occidentale - sous-culture locale, rurale longtemps méconnue - serait donc en cours de réévaluation, de reformulation au début du $\mathrm{xxI}^{\mathrm{e}}$ siècle, dans un contexte interne et international plus favorable. Devenue très marginale, cette tradition reste peu toutefois peu organisée, peu visible et divisée ${ }^{79}$.

\section{Les Pomaques, des musulmans slavophones longtemps dévalués}

Les Pomaques, environ 35000 personnes en Thrace grecque à la fin du $x^{e}$ siècle $^{80}$, constituent des populations charnières, tant en Bulgarie qu'en Grèce ${ }^{81}$. Ces communautés slavophones et musulmanes sont le reflet de brassages culturels, de conversions de chrétiens à l'époque ottomane ${ }^{82}$. À partir de la fin XIX siècle, ces populations - alors fort identifiées à l'islam - sont incorporées aux États-nations. Ces 
États (Bulgarie, Grèce puis Yougoslavie et Turquie) les ont perçues à la fois dans une logique d'inclusion et d'exclusion.

Jusqu'aux années 1970 au moins, ces populations ont été envisagées, en Grèce, au regard de la "menace bulgare ${ }^{83}:$ la Thrace occidentale ayant été sous administration bulgare au Xx siècle - de 1913 à 1920, puis de 1941 à 1944 - l'une des phobies de l'État a longtemps été de contrôler et d'isoler des populations hautement "suspectes $»^{84}$.

31 La politique éducative des musulmans de Thrace grecque a donc d'abord consolidé ces objectifs, unifiant la "minorité » au sein d'un système primaire et secondaire communautaire optionnel, réservé aux seuls musulmans de la région: ces écoles, contrôlées par l'État, dispensent un enseignement en grec et en turc. Le poids de cette seconde langue s'est donc, au total, renforcé au $\mathrm{xx}^{\mathrm{e}}$ siècle parmi les musulmans. Parallèlement, la langue et l'identité slaves/pomaques, d'abord réprimées, ont ensuite été fortement dévalorisées ${ }^{85}$. À partir des années 1950, pour ces populations, l'accès à la langue et à la culture turques représente un vecteur de mobilité, de promotion sociale $^{86}$. L'isolement, le maintien de normes traditionnelles, la ségrégation spatiale dans les villages ruraux pomaques ont aussi encouragé une migration vers les villes Xanthi, Komotini, Alexandroúpolis - puis vers la Turquie ${ }^{87}$. Effets de ces mécanismes psycho-sociologiques et de ces politiques, une partie importante de la population pomaque - ou d'origine pomaque - s'est identifiée à la turcité à la fin du $\mathrm{xx}^{\mathrm{e}}$ siècle $^{88}$.

Ces logiques ont été contestées depuis les années 1990 par des hauts fonctionnaires, des hommes politiques, des associations et autres personnalités grecques, qui pointent le danger d'une turcisation accélérée et totale de la minorité ${ }^{89}$. Ces acteurs ont alors encouragé la revitalisation d'une conscience pomaque, y compris en reconsidérant des traditions rurales jusqu'alors méprisées, en suscitant une production sociologique, ethnologique, linguistique, musicologique en mesure de consolider une telle «identité »: une identité spécifique, ni turque, ni bulgare et si possible, proche de l'hellénisme ${ }^{90}$.

33 Ces projets politiques ont donc eu un volet intellectuel et éditorial. Ainsi, défendue par quelques historiens, ethnologues, voire par des ethnobiologistes ${ }^{91}$, a émergé la thèse selon laquelle les Pomaques seraient les descendants d'une tribu thrace antique, les Agriens. Des membres de ce groupe auraient fait partie des armées d'Alexandre le Grand, au IV ${ }^{\mathrm{e}}$ siècle avant Jésus-Christ. Les Pomaques finissent ainsi par être assimilés à une ancienne tribu de culture hellénique, tardivement slavisée et islamisée ${ }^{92}$. Cette thèse doit être confrontée à celle qui prévaut en Bulgarie, selon laquelle les Pomaques sont des Bulgares, quoique leur confession musulmane les ait marginalisés dans l'État-nation aux $\mathrm{XIX}^{\mathrm{e}}-\mathrm{XX}^{\mathrm{e}}$ siècles $^{93}$. Selon les nationalistes turcs, les Pomaques seraient bien évidemment des Turcs ayant appris la langue slavo-bulgare au contact de populations locales : l'identité religieuse est alors privilégiée et l'ancienne identification entre les termes de "turc » et de «musulman » est opportunément valorisée $e^{94}$.

34 Confrontés à ces versions concurrentes, les Pomaques tendent à articuler ces thèses au seuil du xxI ${ }^{e}$ siècle ${ }^{95}$. Certains, épaulés par des associations pomaco-hellénisantes ont revalorisé leur conscience pomaque, critiquant ouvertement les stratégies expansionnistes turques ${ }^{96}$. D'autres se présentent comme des Turcs: en septembre 2010, le tournage - dans le village de Thermes, au Nord de Xanthi - d'un reportage de la chaîne française FR3 consacré aux Pomaques est violemment perturbé 
par des musulmans locaux, qui affirment haut et fort « ne pas être des Pomaques mais des Turcs $»^{97}$. Les individus peuvent aussi jouer de ces rivalités nationales et de ces mixités identitaires selon les circonstances, selon leur interlocuteur. Parmi bien d'autres, le journaliste Abdulhalım Dede, ancien directeur de la revue turcophone Trakya'nın Sesi (Komotini) se déclare Turc, musulman d'origine pomaque ${ }^{98}$. Rappelant à quel point l'identité pomaque a longtemps été réprimée, il précise : « en fonction de mon intérêt, je me déclare Pomaque ou Turc ${ }^{99}$. Cet aveu - celui d'un intellectuel urbain de la minorité - traduit les ambiguïtés, l'inconfort et dans certains cas, les ajustements identitaires des membres d'un groupe longtemps stigmatisé, tiraillé et instrumentalisé par plusieurs nationalismes ${ }^{100}$.

Les enjeux symboliques cristallisés autour de ces identités minoritaires contribuent à éclairer des attitudes d'incertitude, d'affirmations ostensibles, des logiques contraires, aujourd'hui à l'œuvre: tendances à l'assimilation à des groupes religieux/nationaux plus puissants et réactivation de solidarités internationales.

Depuis les années 1990, l'ouverture de frontières en Europe orientale et balkanique, la revalorisation des «cultures minoritaires " ${ }^{101}$, les mobilisations alévies, le double mouvement de sécularisation et de renouveau religieux ${ }^{102}$ ont eu un impact, y compris dans les villages montagneux de Thrace.

Une enquête de terrain confirme donc ces tendances en apparence contradictoires: ouverture et «modernisation »- des techniques, des outils professionnels, des modes de communication ; élévation générale du niveau d'instruction, gestion peut-être moins problématique des mixités identitaires; rapprochements avec l'islam dominant, facilités par des mariages avec des conjoints sunnites ; migrations ${ }^{103}$; sécularisation des modes de vie; développement de réseaux interétatiques ${ }^{104}$; revitalisation, "folklorisation", médiatisation accrue de fêtes bektachies, non sans immixtion du politique ${ }^{105}$.

Un litige scolaire survenu au cours des années 2000 met précisément en relief ces recompositions subtiles, cet écheveau de logiques sociales et culturelles.

\section{Alévisme, christianisme, hellénisme : discours intellectuelles et pratiques pédagogiques}

\section{Alévisme, christianisme, hellénisme en milieu scolaire : l'affaire Chara Nikopoulou (2004-2010)}

Chronique d'une crise scolaire en milieu alévi

En 2004, une jeune institutrice grecque originaire de Salonique (Macédoine) demande son affectation dans l'école minoritaire de Megalo Dereio/Büyük Derbent ${ }^{106}$. Chara Nikopoulou, par ailleurs fille du président de la Cour de cassation, s'installe avec son époux dans ce village, y transférant y compris ses droits électoraux ${ }^{107}$. Elle se heurte rapidement à la communauté locale, à une partie des parents d'élèves et à la direction de l'établissement.

Le village de montagne est situé au nord du nome grec de l'Évros, à proximité des frontières bulgare et turque ${ }^{108}$. Y résident environ 1000 habitants, principalement alévis. Un tekke subsiste, situé près du village voisin de Mikro Dereio/Küçük Derbent, 
aujourd'hui mixte, chrétien et musulman. La localité s'inscrirait aussi dans un espace de culture pomaque ${ }^{109}$. Mikro Dereio/Küçük Derbent, fondé par les Ottomans au $\mathrm{XIV}^{\mathrm{e}}$ siècle, aurait été essentiellement bulgare " exarchiste " à la fin du XIX siècle et au début du $\mathrm{xx}^{\mathrm{e}}$ siècle ${ }^{110}$.

41 À partir de l'automne 2007, le départ de l'enseignante est régulièrement sollicité, une requête appuyée par des personnalités locales, surtout musulmanes ${ }^{111}$. Cette demande est relayée à l'échelle nationale par le député socialiste (PASOK) musulman de Komotini, Ahmet Hadjiosman ${ }^{112}$. Les tensions culminent avec l'agression physique dont est victime l'institutrice en février 2008. Frappée par un musulman "turciste", circulant ensuite pendant plusieurs mois le bras bandé, Chara Nikopoulou acquiert une notoriété locale et nationale : «bête noire " pour certains, héroïne et martyr pour d'autres ${ }^{113}$.

42 Maintenue à son poste en Thrace durant le mandat du parti conservateur Nea Dimokratia, l'enseignante est mutée à Salonique à l'automne 2010, après la victoire électorale du parti socialiste : cette mutation peut autant se lire comme éloignement ou comme promotion ${ }^{114}$.

\section{Les arguments d'une mission patriotique et éducative}

Durant ces six ans d'exercice à Mikro Dereio, les motifs de litige ont, en fait, été variés. Source incontestable de frictions, l'institutrice s'est investie d'une mission patriotique en terrain scolaire ${ }^{115}$. Renforcer la conscience grecque d'enfants par trop exposés à la "propagande turque» s'inscrit dans un projet pédagogique plus large ${ }^{116}$. Reconnue pour son dynamisme, sa créativité, l'enseignante met entre autres sur pied une chorale, des représentations théâtrales, musicales, valorisant les fêtes nationales grecques, organisant des défilés au cours desquels sont récités poèmes et chansons. Ces morceaux mettent surtout en valeur une identité "helléno-orthodoxe" allant jusqu'à des chansons ouvertement anti-turques ${ }^{117}$. Il s'agit, pour l'institutrice, d'un combat tant éducatif que national. L'enseignante déplore la turcisation et la "kémalisation " des jeunes du village: l'intériorisation par ces enfants des valeurs - plutôt laïques et surtout nationalistes - diffusées en République turque après $1923^{118}$.

De 2004 à 2010, Chara Nikopoulou justifie ses méthodes à Mikro Dereio en soulignant d'une part que ses élèves sont des Pomaques: il s'agirait donc de "Grecs », qui n'auraient «rien à voir avec les Turcs $»^{119}$. D'autre part, les musulmans du village seraient alévis, relevant, par conséquent, d'une tout autre culture, éloignée du sunnisme privilégié par l'État turc ${ }^{120}$. Chara Nikopoulou confie aux journalistes qu'elle a passé du temps avec les parents d'élèves, notamment les mères, qu'elle a beaucoup appris à leur contact, qu'elle a cherché à mieux connaître, à valoriser les coutumes, les fêtes alévies et pomaques. Elle souligne que les familles alévies de son école l'appréciaient, que ses élèves étaient bien "plus Grecs que nombre de chrétiens $»^{121}$. L'institutrice tient à bien dissocier, dans le village, des personnes hostiles voire «fanatiques" - manipulées par le Consulat turc de Komotini - et les musulmans soutenant son travail, spécialement les Alévis. Elle admet qu'en cour de récréation, ses élèves parlent « exclusivement le turc $»^{122}$.

Ses détracteurs musulmans lui reprochent d'être une nationaliste grecque avant d'être une enseignante; d'utiliser ses élèves, l'école pour mener un combat essentiellement politique; de viser une carrière nationale sous couvert de pédagogie appliquée; de 
semer « artificiellement » la discorde dans un village musulman jusqu'alors paisible, de créer une division entre sunnites et alévis locaux ${ }^{123}$. Pour une part de la presse hellénophone, des résidents chrétiens de Komotini, d'Alexandroúpolis, de Xanthi,

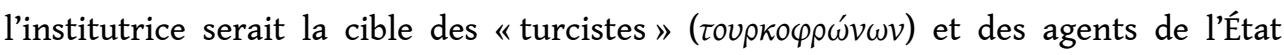
turc $^{124}$. L'attitude de l'institutrice est pourtant désapprouvée par des chrétiens, qui l'accusent de nier la "différence religieuse " de ses élèves, de mener une bataille d'arrière-garde, de miner le travail - moins tapageur, mais peut-être davantage intégrateur - d'enseignants grecs en poste en Thrace depuis des années ${ }^{125}$.

Chara Nikopoulou a aussi ses laudateurs, nombreux en Thrace et, à l'échelle du pays, dans la mouvance patriotique : Grecs au profil patriotique classique, modéré, mais aussi nationalistes au discours plus agressif et radical ${ }^{126}$. Les formations d'extrême droite

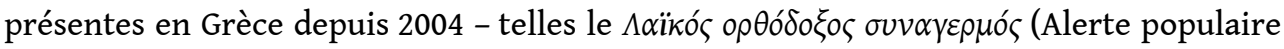
orthodoxe, LAOS) et X $\rho v \sigma \eta ́$ Avүŕ (Aube dorée) - se sont saisies du dossier, apportant leur soutien à une enseignante « modèle ». À l'échelle locale, des musulmans pomaques - et "pomacistes ", proches du journal Zagalisa - valorisent l'institutrice, sa bienveillance à l'égard du « combat » pomaque de Thrace ${ }^{127}$.

L'affaire Chara Nikopoulou n'a pas simplement soudé les communautés musulmanes de Thrace contre un ennemi, un « corps étranger ", femme de surcroît, venue de Salonique et non du cru régional, un facteur de troubles pour les villages de Thrace grecque. Le litige a parallèlement réactivé des lignes de clivages - multiples et anciennes - au sein de la minorité. Témoin de rivalités internes, les échanges virulents entre deux journalistes musulmans, Cengiz Ömer - qui écrit dans le Millet de Xanthi - et Ilhan Tahsin - du journal Birlik, de Komotini. Ömer, lui-même natif d'un village pomaque de Thrace, Glavki, accuse son collègue de ne pas briller pour sa piété et de défendre la cause turco-musulmane essentiellement par opportunisme ${ }^{128}$.

Ces accusations en disent long sur des divisions toujours vivaces parmi les «musulmans» de Thrace, entre des personnes restées attachées aux traditions religieuses et des laïcs, très critiques à l'égard des rites et pouvoirs religieux, survalorisant la figure tutélaire de Mustafa Kemal. Ces clivages internes se situent aussi à l'échelon identitaire, entre les deux pôles principaux des "turcistes" et des « pomacistes » militants. Enfin, des frontières subtiles, mais bien ancrées entre sunnites et alévis/bektachis sont réactivées de 2004 à 2010, à travers ce litige scolaire. En juillet 2010 et mai 2011, ils n'ont pas été rares les musulmans de Thrace qui, interrogés sur la présence bektachie dans la région baissent la voix, précisant qu'eux-mêmes « ne fréquentent pas les Alévis » ou indiquant que les Alévis ne sont « pas véritablement des musulmans $»^{129}$.

Si Mme Nikopoulou met en avant l'identité pomaque du village de Mikro Dereio, les archives de l'État suggèrent une composition démographique un peu plus mêlée, du moins dans les années 1960. Ainsi, le Conseil Consultatif de Thrace, dans un rapport daté du 23 juillet 1964 mentionné par l'historien Tásos Kōstópoulos recense « 64 familles turques et 235 familles pomaques » dans les deux villages de Sidirochori et de Megalo Dereio ${ }^{130}$. Selon ce même rapport, la plupart des familles pomaques concernées, toutes issues du nome de Rhodope, se seraient installées dans ces villages de « 1942 à $1955 »^{131}$. 
L'école, un théâtre stratégique : islam et éducation en Grèce aux $x x^{e}-x^{e} I^{e}$ siècles

50 Ces tensions locales - aux plus larges ramifications ${ }^{132}$ - confirment le caractère stratégique de l'institution scolaire dans cette région multicommunautaire. Depuis 25 ans, une grande partie des travaux sociologiques de langue grecque sur la Thrace concernent l'éducation: ce secteur représente effectivement un enjeu majeur dans cette région, ouvrant d'ailleurs une réflexion pédagogique plus profonde et novatrice dans un pays ayant redécouvert et renforcé sa dimension pluriculturelle ${ }^{133}$.

51 La durée du litige de Megalo Dereio a reflété la posture hésitante du ministère grec de l'Éducation nationale et des Cultes, ses divergences internes. Quels sont les critères permettant de définir un bon enseignant, notamment en milieu pluriculturel? L'article 16 de la Constitution place «le développement d'une conscience nationale et religieuse " parmi les missions de l'Éducation nationale en Grèce ${ }^{134}$. Cette clause a été invoquée par Chara Nikopoulou, qui insiste sur le caractère légal et légitime de sa démarche. Primée par l'Académie d'Athènes ${ }^{135}$, par la préfecture de Salonique ${ }^{136}$, Chara Nikopoulou est encensée par des acteurs patriotes ${ }^{137}$.

52 Un autre document juridique régit pourtant les liens entre éducation et islam en Thrace depuis 1923 : le Traité de Lausanne, associé à des conventions bilatérales grécoturques. Or, ce texte garantit - et peut-être en la réifiant un peu trop ? - «l'identité » particulière des musulmans de la région ${ }^{138}$. L'institutrice est donc aussi l'objet de vives critiques y compris au sommet du Ministère. De 2009 à l'automne 2010, au cœur du litige de Megalo Dereio, c'est en effet une universitaire, Thalia Dragona, qui occupe le poste de Secrétaire spéciale chargée des Programmes au ministère de l'Éducation nationale ${ }^{139}$. Or, cette enseignante de l'Université Capodistria d'Athènes maitrise tout particulièrement les enjeux éducatifs en milieu musulman.

Professeur de psychologie sociale, sociologue de l'éducation, Thalia Dragona a en effet accru sa notoriété en coéditant un ouvrage collectif qui marque la Grèce des années 1990 : l'ouvrage met l'accent sur les logiques «ethnocentristes» qui soustendraient alors une grande partie des manuels scolaires grecs ${ }^{140}$. Spécialiste des enjeux pédagogiques en espace multiculturel, Dragona fut précisément l'une des coordinatrices d'un «Programme d'enseignement pour les élèves musulmans de Thrace », programme mis en œuvre depuis l'année 1997-1998 ${ }^{141}$.

Les politiques étatiques à l'égard des musulmans de Thrace ont en effet connu une évolution depuis le milieu des années 1990. Ce tournant, qui suit une décennie d'exacerbation identitaire et nationaliste dans la région ${ }^{142}$, est particulièrement perceptible dans ce domaine de l'éducation avec un projet explicite - et alors inédit de pleine intégration des jeunes musulmans dans la société grecque ${ }^{143}$. Le Programme d'enseignement aux élèves musulmans (PEM) participe de cette nouvelle approche. Il s'agirait de briser le retard, "l'exclusion", la «marginalisation" des jeunes musulmans. Il s'agit aussi d'améliorer la maîtrise du grec - gage d'une mobilité accrue au sein de la société hellénique - tout en reconnaissant à l'école les spécificités culturelles, linguistiques, religieuses de ces élèves ${ }^{144}$. Le pomaque n'est pas repris comme langue du système éducatif minoritaire. L'activité de Chara Nikopoulou à Mikro Derio a pour contexte cette réforme, appliquée depuis plusieurs années ${ }^{145}$.

La démarche de Mme Nikopoulou a aussi trouvé des appuis dans une constellation de travaux de sciences sociales qui, prolongeant une problématique marquante parmi les orientalistes européens des $\mathrm{XIX}^{\mathrm{e}}-\mathrm{XX}{ }^{\mathrm{e}}$ siècles, tend à envisager l'alévisme-bektachisme 
comme le produit d'influences «extérieures à l'islam », spécialement du monachisme chrétien ${ }^{146}$.

\section{Regards sur l'islam hétérodoxe, entre jugement normatif, sciences sociales et discours nationaux}

\section{Un courant longtemps ignoré et repoussé dans l'altérité}

Dans la bibliographie hellénophone en sciences sociales, l'alévisme n'a certes pas représenté un thème marquant au $\mathrm{xx}^{\mathrm{e}}$ siècle: les alévis, parce qu'inscrits dans l'ensemble musulman, parce que très peu nombreux dans le pays, ont été le plus souvent ignorés ou envisagés comme membres d'une autre religion ${ }^{147}$.

Pourtant, comme le pointe l'historien Michalis Kokolakis, après la Première Guerre mondiale, plusieurs textes nationalistes grecs consacrés à l'Asie Mineure mentionnent les Kizilbash et cette fois, pour les différencier des «musulmans» et des «Turcs » ${ }^{148}$. Dans une conférence publiée en 1921, Konstantinos Lameras inclut même ce groupe dans la catégorie des "crypto-chrétiens ${ }^{149}$. Dans ce "nouveau » regard sur l'islam hétérodoxe d'Anatolie ${ }^{150}$, est perceptible l'influence de chercheurs, d'orientalistes européens, de missionnaires qui se penchent, au tournant des XIX-XXe siècles, sur les liens entre islam et christianisme. C'est le cas de l'archéologue britannique Frederick Hasluck, célèbre pour ses travaux menés en Anatolie et dans les Balkans au début du $\mathrm{Xx}^{\mathrm{e}}$ siècle ${ }^{151}$. Toutefois, se sont ajoutés des enjeux politiques plus concrets : à la fin de la Première Guerre mondiale, l'annexion par la Grèce d'une partie occidentale de l'Anatolie semble plus que jamais envisageable. Rapprocher les musulmans hétérodoxes de la famille chrétienne ne permettait-il pas de mieux justifier l'incorporation d'une portion de cette région à la patrie? Cette thèse n'est néanmoins ni officielle ni dominante au début des années $1920^{152}$.

\section{Nos proches méconnus : islam hétérodoxe et christianisme orthodoxe}

Depuis les années 1980, la question de l'islam hétérodoxe réapparaît dans les sciences sociales de langue grecque: après l'étude importante d'Evstratios Zegginis ${ }^{153}$ datée de 1988, le thème est abordé par des anthropologues, sociologues, folkloristes, et plus récemment, des ethnomusicologues ${ }^{154}$. Une partie non négligeable de ces travaux dont l'ouvrage de Zegginis - reprend cette problématique des proximités entre

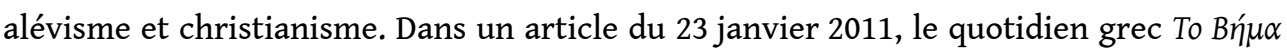
(La Tribune) titrait ainsi, de manière intéressante: «Les alévis, nos proches méconnus $»^{155}$.

Ainsi, les différences vis-à-vis du sunnisme sont d'autant soulignées: usage, dans l'alévisme, de lieux de culte spécifiques; observation non obligatoire ou moins longue du jeûne de ramadan ; absence de séparation rigoureuse entre hommes et femmes lors des cérémonies religieuses. Symétriquement, les proximités entre alévisme et orthodoxie sont mises en relief: usage du vin, des bougies; culte des saints; monogamie. Le triptyque Allah-Mohamet-Ali ne renverrait-il pas à la Trinité chrétienne; la valorisation du chiffre 12 ne rappellerait-elle pas les 12 Apôtres du Christ? L'accent placé sur le pèlerinage du cœur, sur l'amour de Dieu et du prochain sont alors autant de points communs. Le cem, les cérémonies initiatiques deviennent alors des sortes de baptêmes ou de communions, je reprends ici l'analyse de Zegginis, 
auteur de l'ouvrage le plus complet sur la question ${ }^{156}$. Ces similarités apparaissent alors comme preuves d'une trace primordiale et décisive du christianisme ${ }^{157}$.

Ces thèses sont exprimées de manière plus directe encore par Dimitris Kitsikis, professeur de relations internationales à l'Université d'Ottowa. Dans un ouvrage paru en 2000 et intitulé l'Importance du bektachisme-alévisme pour l'hellénisme, l'auteur écrit :

L'avenir de l'espace helléno-turc dépend des alévis (...) Grâce au bektachisme, l'idéologie des Janissaires, élaborée par l'Empire ottoman dans la Région intermédiaire ${ }^{158}$ est restée aux mains des Romioi, c'est-à-dire des Grecs chrétiens orthodoxes (...). Sans l'alévisme, la Turquie s'éloignera de la Grèce et basculera dans les bras de l'islamisme arabe. Avec les Alévis, l'État helléno-turc sera religieux, mais tolérant et les valeurs de l'hellénisme - communes aux Grecs et aux Turcs prévaudront ${ }^{159}$.

61 Cet ouvrage se pose d'abord comme travail scientifique, l'auteur se définit lui-même comme turcologue. Toutefois, Kitsikis se présente aussi comme un acteur, soulignant sa sympathie à l'égard de la religion alévie, une attitude positive qui transparaît dans plusieurs travaux sur ce courant de l'islam. Valorisé par nombre d'auteurs occidentaux, l'islam hétérodoxe semble alors érigé en un bon islam ${ }^{160}$.

Panagiotis Karakatsanis, professeur à l'Université Dimokriteio de Thrace, spécialiste de pédagogie, insiste, quant à lui, sur la continuité directe entre le bektachisme et l'orphisme, ce mouvement mystique et dionysiaque de la Grèce antique. Cette analyse le conduit à éloigner l'alévisme de Thrace de l'islam et du monde turc pour le replacer dans la continuité de croyances locales autochtones et de la culture hellénique ${ }^{161}$. Selon des lectures plus diffuses, les bektachis - de même que les Pomaques - seraient les descendants de plus anciennes hérésies chrétiennes byzantines: paulicianisme et bogomilisme, deux courants dualistes, ayant développé un discours de critique du pouvoir ${ }^{162}$. Le paulicianisme est un mouvement religieux chrétien oriental qui s'est développé dans l'Empire byzantin - en Anatolie - au VII siècle de notre ère. Ce mouvement aurait inspiré le bogomilisme, courant dualiste qui émerge à partir du $\mathrm{x}^{\mathrm{e}}$ siècle en Bulgarie, en Serbie, en Bosnie et plus généralement dans la péninsule balkanique. La Thrace aurait constitué un terrain précurseur de prédication bogomile ${ }^{163}$.

63 Ces analyses intellectuelles ont pu fournir des éléments, des arguments, qui éclairent ce conflit scolaire. Le discours de Chara Nikopoulou de 2004 à 2010 s'est appuyé sur un socle intellectuel.

\section{Conclusion}

Longtemps rejeté hors de l'idée nationale, l'islam reste une question sensible en Grèce, devenue pays d'immigration et l'une des principales portes d'entrée dans l'Union européenne ${ }^{164}$. Si aujourd'hui la majorité des musulmans de Grèce résident en Attique et dans la région de Salonique, l'islam turcophone et bulgarophone de Thrace a toujours représenté au $\mathrm{xx}^{\mathrm{e}}$ siècle un enjeu politique. Ceci a valu y compris pour l'alévisme, un courant ancien, affaibli, écartelé aujourd'hui entre effacement, assimilation au sunnisme, reconstruction de réseaux transfrontaliers et revitalisation identitaire ${ }^{165}$. Le conflit scolaire de Megalo Dereio constitue à cet égard un exemple extrême par son caractère accusé, clairement formulé et surtout médiatisé ${ }^{166}$. La démarche de la protagoniste du litige met pourtant en relief les paradoxes des 
mouvances nationalistes grecques : au seuil du xxI ${ }^{\mathrm{e}}$ siècle, faut-il nationaliser ou exclure les musulmans de la nation? Le projet de l'institutrice s'est heurté à un système de pouvoirs locaux et à la force d'identités nationales/culturelles concurrentes. La résistance d'une grande partie de la population a-t-elle signifié que pour ces habitants, l'alévisme ne pouvait être réduit à un "crypto-christianisme », un crypto-hellénisme ? Ce conflit a aussi représenté un terrain fertile pour l'extrême droite, en essor en Grèce depuis les années $2000^{167}$.

Cette affaire souligne à quel point l'alévisme/bektachisme se trouve confronté aux identités dominantes - nationales ou religieuses ${ }^{168}$. Le litige de Megalo Dereio, qui articule logiques locales, nationales et inter-étatiques, montre les tensions cristallisées, dans les Balkans contemporains, autour de l'islam hétérodoxe.

\section{BIBLIOGRAPHIE}

\section{Bibliographie sélective}

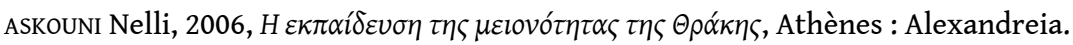

BALIVET Michel, 1992, « Aux origines de l'islamisation des Balkans », Revue des mondes musulmans et de la Méditerranée 66, p. 10-22.

BOZARSLAN Hamit, 2007, Histoire de la Turquie contemporaine, Paris : La Découverte.

BRAUDE Benjamin, LEWIS Bernard (eds.), 1982, Christians and Jews in the Ottoman Empire: The Functioning of a Plural Society, New York-Londres: Holmes and Meier.

CLAYER Nathalie, BOUgARel Xavier, 2013, les Musulmans d'Europe du Sud-Est, Paris : Karthala. DALÈGRE Joëlle, 1997, la Thrace grecque : populations et territoires, Paris : L'Harmattan.

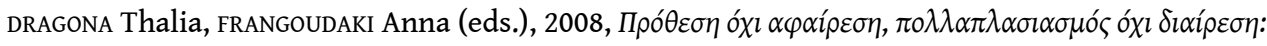

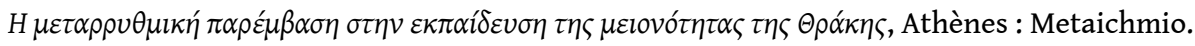

HASLUCK Frederick, 1929, Christianity and Islam under the Sultans, Oxford: Oxford University Press, 2 volumes.

HERSANT Jeanne, 2008-2009, « Surveillances croisées et rivalité gréco-turque en Thrace occidentale », European Journal of Turkish Studies 8, article en ligne, https://ejts.revues.org/2693

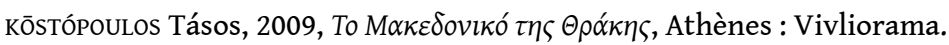

LAMPE John, MAZOWER Mark (eds.), 2004, Ideologies and National Identities in Southeastern Europe, New York: Central Europe University Press.

LORY Bernard, 2005, les Balkans : de la transition post-ottomane à la transition post-communiste, Istanbul : ISIS. 
LOWRY Heath, 2009, In the Footsteps of the Ottomans: A Search for Sacred Spaces and Monuments in Northern Greece, Istanbul: Bahçeşehir Université.

MASSICARD Élise, 2005, l'Autre Turquie : le mouvement aléviste et ses territoires, Paris : PUF.

MAVRommatis Giorgos, 2003, "Constructing Identities for the Thracian Muslim Youth: the Role of Education", Jahrbücher für Geschichte und Kultur Südosteuropas 5, pp. 113-123.

MAVROMMATIS Giorgos, 2008, "Bektachis in the xxth Century Greece”, Turcica 40, pp. 243-245.

MAZOWER Mark, 2000, The Balkans, Londres: Weidenfeld and Nicholson.

MÉLIKOFF Irène, 1992, Sur les traces du soufisme turc : recherches sur l'islam populaire en Anatolie, Istanbul : ISIS.

MÉLIKOFF Irène, 2001, Au banquet des quarante : exploration au cœur du Bektachisme-Alévisme, Istanbul : ISIS.

NEUBERGER Mary, 2004, The Orient Within: Muslim Minorities and the Negociation of Nationhood in Modern Bulgaria, Ithaca/Londres: Cornel University Press.

POPOVIĆ Alexandre, VEINSTEIN Gilles, (dir.), 1996, les Voies d'Allah : les ordres mystiques dans l'islam des origines à aujourd'hui, Paris : Fayard.

STEINKE Klaus, Voss Christian (eds.), 2007, The Pomaks in Greece and in Bulgaria, Munich: Verlag.

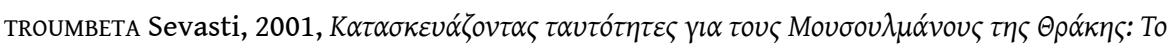

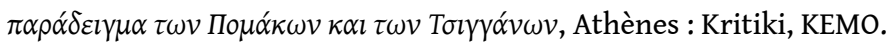

TSIBIRIDOU Fotini, 2000, les Pomaks dans la Thrace grecque : discours ethniques et pratiques socioculturelles, Paris : L'Harmattan.

TSITSELIKIS Konstantinos, 2012, Old and New Islam in Greece, Leiden-Boston: Martinus Nijhoff. VEINSTEIN Gilles (dir.), 2005, Syncrétismes et hérésies dans l'Orient seldjoukide et ottoman (XIVXVIII ${ }^{e}$ siècles), Bruxelles : Peeters.

ZARCONE Thierry, 2009, le Soufisme : voie mystique de l'islam, Paris : Gallimard.

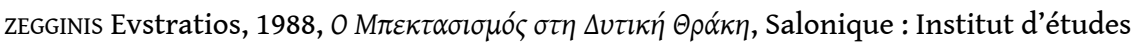
balkaniques.

\section{NOTES}

1. Conversation avec l'auteur, village de Roussa/Ruşanlar, 6 mai 2011.

2. Xavier bougarel, Nathalie Clayer, 2013, les Musulmans de l'Europe du Sud-Est. Plus

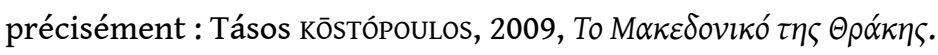

3. http://www.uoc.edu/euromosaic/web/homefr/index1.html.

4. Daniel PANZAC (dir.), 1992, les Balkans à l'époque ottomane, numéro spécial de la Revue du monde musulman et de la Méditerranée 66, (4); Benjamin BRAUDE, Bernard LEWIS (eds.), 1982, Christians and Jews in the Ottoman Empire; Christine wOODHEAD (ed.), 2012, The Ottoman World, New York: Routledge ; Richard HALL, 2011, The Modern Balkans : A History, Londres: Reaktion Books. 
5. Mark MAZOWER, 2000, The Balkans; Hamit BOZARSLAN, 2007, Histoire de la Turquie contemporaine; Meropi ANASTASSIADOU, 1997, Salonique: une ville ottomane à l'âge des Réformes 1830-1912, Leiden: Brill; John LAMPE, Mark MAZOWER (eds.), 2004, Ideologies and National Identities in Southeastern Europe.

6. Étienne COPEAUX, 1997, Espace et temps de la nation turque, Paris: CNRS éditions ; Sabrina RAMET, 1998, Nihil Obstat, Religion, Politics and Social Change and Eastern Europe and Russia, Durham: Duke University Press ; Bernard LORY, 2005, les Balkans : de la transition post-ottomane à la transition post-communiste ; Emma AUBIN-BOLTANSKI, 2007, Pèlerinages et nationalisme en Palestine: prophètes, héros, ancêtres, Paris: éditions de l'EHESS; Sossie ANDÉZIAN, 2012, le Sacré à l'épreuve du politique : Noël à Bethléem, Paris : Riveneuve éditions.

7. Kristina KOULOURI (ed.), 2002, Clio in the Balkans: The Politics of History Education, Thessalonique: CDRSEE; Pierre NORA (dir.), 1984-1992, les Lieux de mémoire, Paris : Gallimard, volumes 1-3; Jean-François CHANET, 1996, l'École républicaine et les Petites Patries, Paris : Aubier ; Anne-Marie THIESSE, 1999, la Création des identités nationales, Paris : Seuil.

8. Mary NEUBERGER, 2004, The Orient Within: Muslim Minorities and the Negociation of Nationhood in Modern Bulgaria.

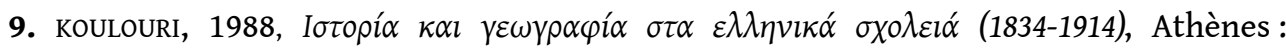
Archives historiques de la jeunesse hellénique ; Iannis KOUBOURLIS, 2005, la Formation de l'histoire nationale grecque: l'apport de Spyridon Zambelios (1815-1881), Athènes : IRN/FNRS ; Isabelle DÉPRET, 2009, Église orthodoxe et histoire en Grèce contemporaine : versions officielles et controverses historiographiques, Paris : L'Harmattan ; Michael HERTZFELD, 1982, Ours once more: Folklore, ideology and the Making of Modern Greece, Austin: University of Texas Press.

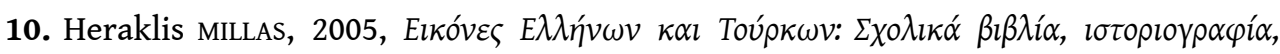

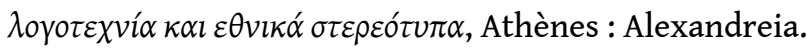

11. Ceci apparaît clairement dans les archives du Centre d'études micrasiatiques d'Athènes.

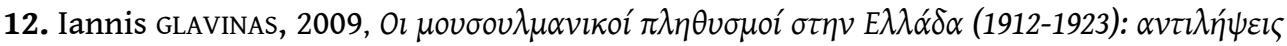

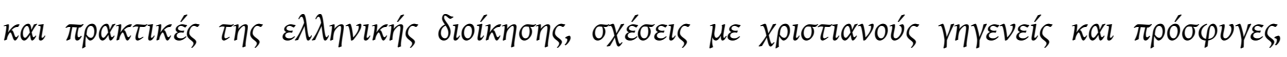
Université Aristote de Salonique, Faculté de Philosophie, Département d'archéologie et d'histoire, p. 26.

13. Nous retrouvons aux $\mathrm{XIX}^{\mathrm{e}}-\mathrm{XX} \mathrm{e}^{\mathrm{e}}$ siècles des mécanismes proches dans d'autres Étatsnations balkaniques tels la Serbie, la Bulgarie, la Roumanie. Entre autres: Sylvie Gangloff (dir.), 2005, la Perception de l'héritage ottoman dans les Balkans, Paris : L'Harmattan.

14. Une haute administration hellénique où les musulmans sont très largement exclus au $\mathrm{XX}^{\mathrm{e}}$ siècle. Konstantinos TSITSELIKIS, 2012, Old and New Islam in Greece, p. 130-138; GLAVINAS, 2009, p. 56-57.

15. Archives du ministère des Affaires étrangères grec AMAEH, 1928, dossier " dédommagements pour des propriétés aliénées »; 1930, dossier A/4/I, "Propriétés albanaises en Grèce »; 1932, dossier B/5/III, "Propriétés nationalisées »; 1935, dossier A/4/9 «Minorité albanaise de Tchamouria ». Également, GLAVINAS, 2009, Ol

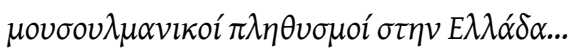




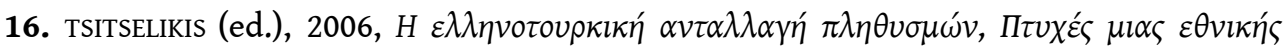

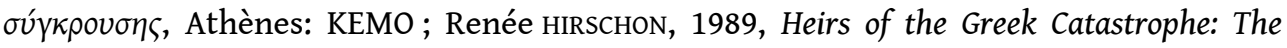
Social Life of Asia Minor Refugees in Piraeus, New York: Oxford University Press; Dimitris PENTAZOPOULOS, 2002, The Balkan Exchange of Minorities and its Impacts on Greece, Londres: Hurst and Company.

17. The Treaties of Peace 1919-1923, Volume II, 1924; Samim AKGÖNÜL, 1999, Une minorité, deux États : la minorité turco-musulmane de Thrace occidentale, Istanbul : Isis.

18. Joëlle DALÈGRE, 1997, la Thrace grecque : populations et territoires, Paris : L'Harmattan.

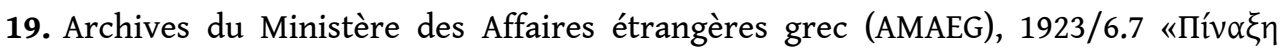

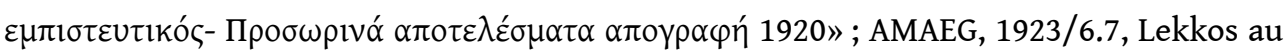
Gouverneur général de Macédoine Salonique, 31 mars 1923, nº 394 et sq. Également, GLAVINAS, 2009, p. 115.

20. "La minorité musulmane de Thrace », Ministère des Affaires étrangères grec, article en ligne: http://www.mfa.gr/; Byron KOTZAMANIS, Michalis AGORASTAKIs, 2006, "La minorité musulmane de Thrace: la mesure du caché », Démographie et culture, p. $442-460$.

21. KOTZAMANIS, AGORASTAKIS, 2006, «La minorité musulmane de Thrace : la mesure du caché », p. 454.

22. Michel BRUNEAU (dir.), 1998, les Grecs pontiques, Paris: CNRS éditions;

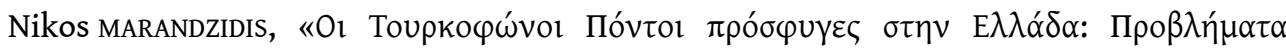

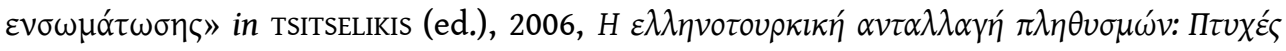

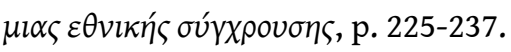

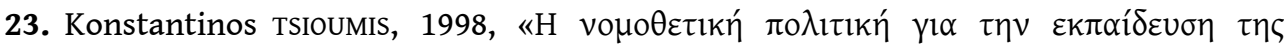

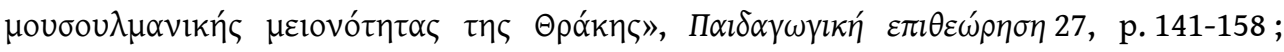

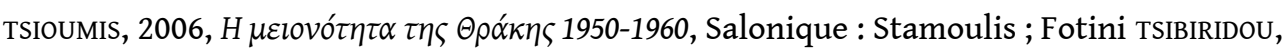
2000, les Pomaks dans la Thrace grecque; Bernard VERNIER, 1998, "Représentation mythique du monde et domination masculine chez les Pomaques grecs ", Actes de la recherche en sciences sociales 125, p. 74-98.

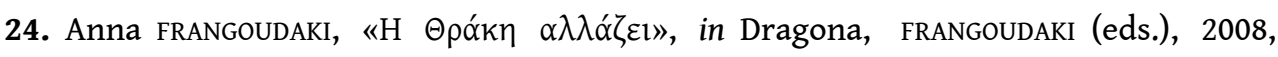

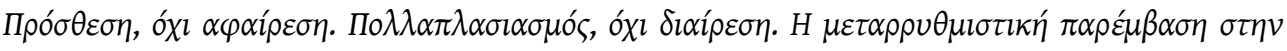

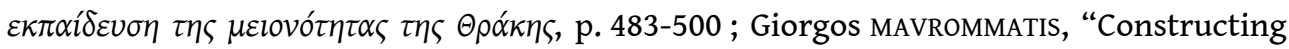
Identities for Thracian Muslim Youth: The Role of Education" in Sevasti TROUMBETA, Christian voss (eds.), 2003, Minorities in Greece: Historical Issues and New Perspectives, numéro spécial, Jahrbücher für Geschichte und Kultur Südosteuropas 5, pp. 113-123.

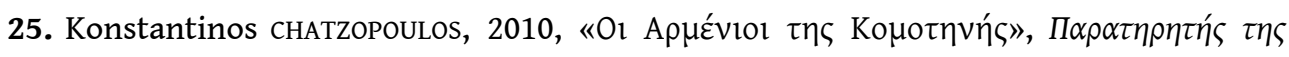

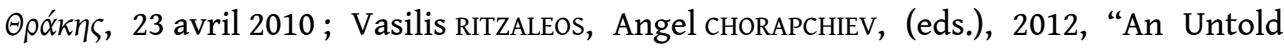
Story: Testimonies on the Salvation of the Greek Jews Mobilized to the Bulgarian Forced Labour Camp in Belitsa (1943)", Atelier Archives of Memory: The Experience of Greek Jews in Audiovisual Testimonies, Université de Volos, 24-25 février 2012.

26. Barbara JELAVIC, 1983, History of the Balkans: xxth Century, Cambridge: Cambridge

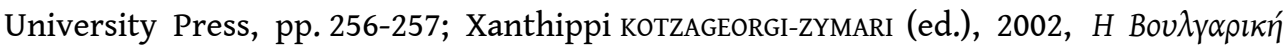

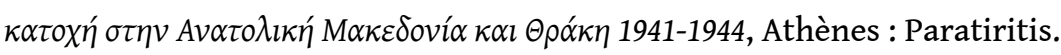

27. Pour le point de vue d'un ottomaniste américain, Heath LowRY, 2009, In the Footsteps of the Ottomans, A Search for Sacred Spaces and Monuments in Northern Greece, chapitre 1. 
Voir également le récent ouvrage édité par le Ministère de la Culture hellénique,

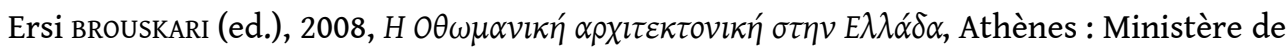
la Culture.

28. Voir ici Galia VAltchinova (ed.), 2010, Religion on the Boundary and the Politics of Divine Interventions, Istanbul: Isis.

29. Depuis les années 1990, il existe aussi deux muftis "élus » par une partie de la communauté musulmane, financés par l'État turc et dont les fonctions sont davantage symboliques et politiques. Sur cette polémique, TSITSELIKIS, 2012, Old and New Islam..., pp. 367-428.

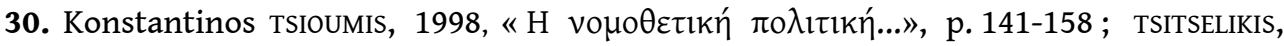

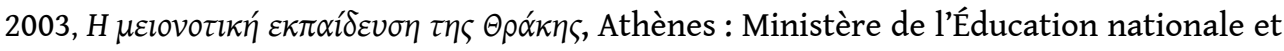

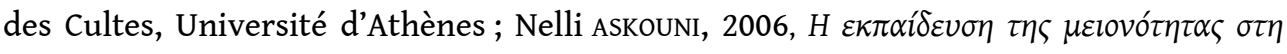

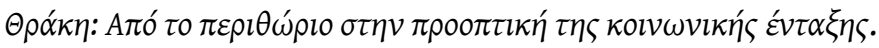

31. «La minorité musulmane de Thrace», http://www.mfa.gr/, consulté le 14 mars 2016.

32. Voir la critique de la thèse syncrétique à propos du soufisme dans Mark SEDGWICK, 2001, le Soufisme, Paris : Cerf, p. 12-13.

33. Irène MÉLIKOFF, 1992, Sur les traces du soufisme turc: recherches sur l'islam populaire en Anatolie, Istanbul : ISIS ; Alexandre POPović, Gilles VEINSTEIN, (dir.), 1996, les Voies d'Allah: les ordres mystiques de l'islam des origines à aujourd'hui; Machiel KIEL, "Ottoman Development and the Cult of a Heterodox Sufi Saint: Sari Saltik Dede and Towns of Isakçe and Babadag in the Northern Dobrudja", in VEINSTEIN (dir.), 2005, Syncrétismes et hérésies dans l'Orient seldjoukide et ottoman (XIV-XVIII' siècles), p. 283-298.

34. MÉLIKOFF, 2001, Au banquet des quarante : exploration au cœur du Bektachisme-Alévisme;

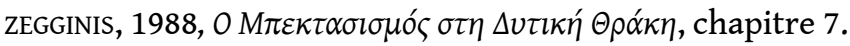

35. Encyclopédie de l'islam, 1960, volume I, Leiden : Brill, p. 1196-1197.

36. Encyclopédie de l'islam, 1960, p. 1196-1197. MÉLIKOFF, 1998, Hadji Bektash, un mythe et ses avatars : genèse et évolution du soufisme en Turquie, Leiden : Brill.

37. MÉLIKOFF, 2001, Au banquet... Pour le cas albanais, Nathalie CLAYER, 1995, "Bektachisme et nationalisme albanais", in POPOVIć, vEINSTEIN (dir.), Bektachiyya : études sur l'ordre mystique des Bektachis et les groupes relevant de Hadji Bektach, Istanbul: Isis, p. 277-308.

38. Éric GEOFFROY, 2004, Initiation au soufisme, Paris: Fayard; Rachida $\mathrm{CH}$, 2000, le Soufisme au quotidien : confréries d'Égypte au XXe siècle, Arles : Sindbad.

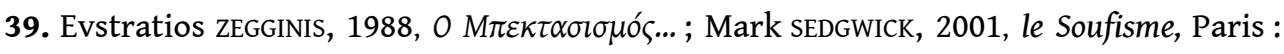
Cerf; Ambrosio Alberto FABIO et alii, 2006, les Derviches tourneurs: doctrine, histoire, pratiques, Paris: Cerf; Sabrina MERVIN, 2006, "Les larmes et le sang des Chiites: pratiques rituelles lors de la célébration de Ashûra (Liban, Syrie) », Revue des mondes musulmans et de la Méditerranée 113-114, p. 153-166.

40. Mehmet Koç, 2011, Seyyid Ali Sultan Dergâhı ve Osmanlı Eserleri, Alexandroupolis: Dunden Bugüne; Riza YILDIRIM, 2010, «Bektaşi Alevi Geleğine göre Seyyid Ali Sultan », Türk kültürü ve Hacı Bektaş Veli Araştırma Dergisi 53, p. 59-88. 


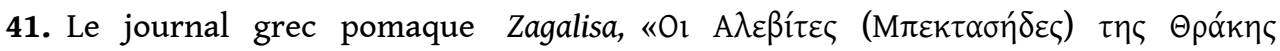

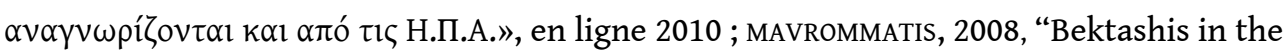
20th Century Greece", Turcica 40, pp. 219-250.

42. Roger SAVORY, "Kizil-Bash", Encyclopaedia of Islam, vol. 3, Leiden: Brill, pp. 243-245; MÉLIKOFF, 1992, Sur les traces du soufisme turc.

43. MÉLIKOFF, 1998, Hadji Bektash, un mythe et ses avatars, p. 20-21; ZEGGINIS, 1998, 0

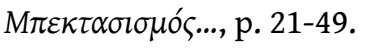

44. John K. BIRGE, 1965, The Bektashi Order of Dervishes, Londres: Luzac, (1933); Alexandre POPOVIĆ, 1994, les Derviches balkaniques, hier et aujourd'hui, Istanbul, Isis ; POPOVIĆ, VEINSTEIN (dir.), 1996, les Voies d'Allah.

45. Suraiya FAROQHI, 1976, “Agricultural Activities in a Bektashi Center: The Tekke of Kizil Deli 1750-1830", Südost Forshungen 35, p. 69-96; Randi DEGUILHEM, 1995, le Waqf dans l'espace islamique: outil de pouvoir socio-politique, Paris: Institut français de Damas -

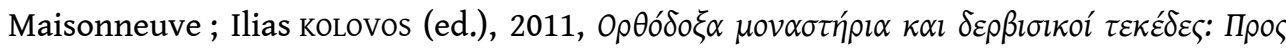

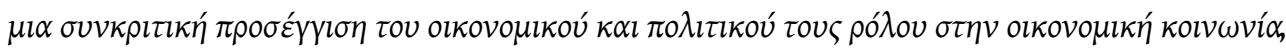
Heraklion : Université de Crète.

46. Au début $d u X V I^{e}$ siècle, les Kizilbash représentent, aux frontières ottomano-perses, des militants influencés par le chiisme ainsi que des partisans des Safavides dans la guerre ottomano-perse. La suppression violente du corps des janissaires en 1826 par le sultan Mahmut II - un corps très lié au bektachisme - et la vente prévue des biens des tekke à cette date ont nettement atteint la puissance de cette confrérie ; Fahr MADEN, 2010, «Kizildeli Sultan tekkesi'nin kapatilması (1826) ve faaliyetlerine yeniden başlaması»", Türk Kültürü ve Hacı Bektaş Veli araştırma dergisi53, p. 115-126; Michel BALIVET, 1992, "Aux origines de l'islamisation des Balkans ", Revue des mondes musulmans et de la Méditerranée 66, p. 10-22 ; MÉLIKOFF, 1983, «L'ordre des Bektaşi après 1826 », Turcica Xv, p. 155-178 ; MAVROMMATIS, 2008, p. 219-250.

47. Les tekke sont des loges/couvents de derviches et lieux de culte hétérodoxes.

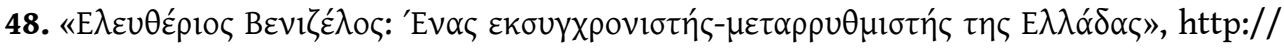

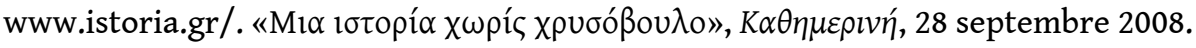

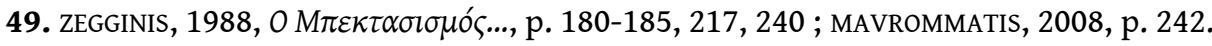

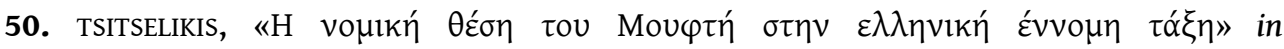

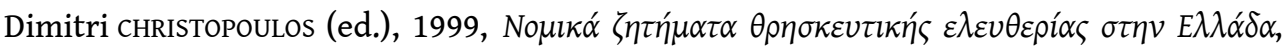

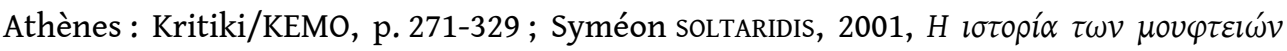
$\tau \eta \varsigma \Delta v \tau i \kappa \eta ́ s ~ \Theta \rho \alpha ́ \kappa \eta \varsigma$, Athènes.

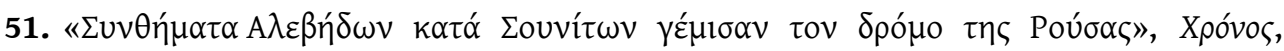
28 juin 2008.

52. Entretiens, mai 2011.

53. Entretiens, mai 2011.

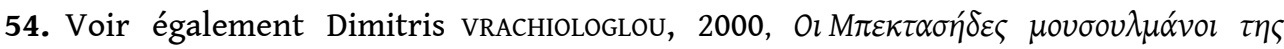

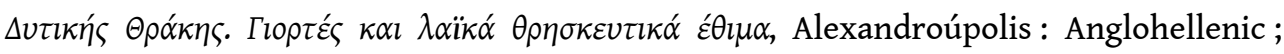
Miranda TERZOPOULOU, 2009, communication "The Evolution of a Bektashi Panayir in Greek Thrace", Conférence internationale consacrée à Haci Bektash Veli, Ankara, 19-21 octobre. 
55. Observations et entretiens réalisés en juillet 2010 et mai 2011.

56. Fotini TSIBIRIDOU, 2000, les Pomaks..., notamment le chapitre 3, p. 141-184;

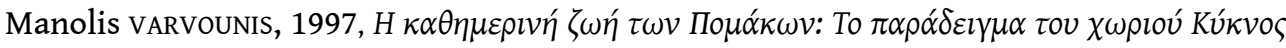
$\tau \eta \varsigma \Xi \alpha ́ v \theta \eta \zeta$, Athènes : Odysseas.

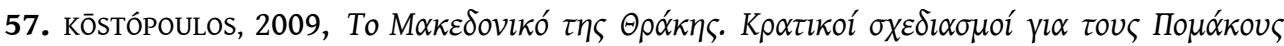
1956-2008, Athènes : Bibliorama, 2009 ; Katerina MARKou, 2002, « Les Pomaques de Thrace grecque et leurs choix langagiers ", Études balkaniques 9, p. 41-52.

58. Entretiens, juillet, septembre 2010.

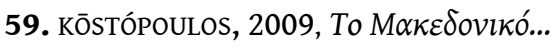

60. Entretiens menés au Nord de la Thrace grecque, mai 2011.

61. Célébré dans les villages alévis du Nord de la Thrace grecque, mais aussi parmi les Tziganes. Également John BIRGE, 1965, The Bektashi, p. 39-40; MÉLIKOFF, 1998, Hadji Bektach..., p. 180 ; Maria CouroucLI, "Saint Georges l'Anatolien, maître des frontières ", in Maria couroucl, Dionigi albera (dir.), 2009, Religions traversées, Paris: Actes Sud, p. $175-208$.

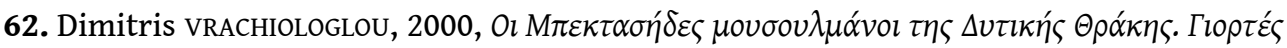

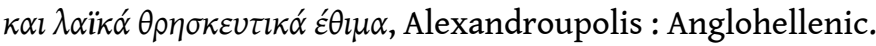

63. Ceci vaut pour les festivités estivales de Setchek, sur le plateau de Chilia, festivités qui semblent avoir acquis une dimension folklorique, touristique et politique. Entre

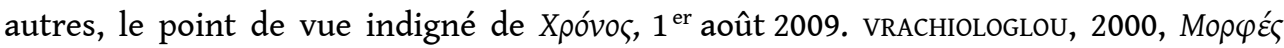

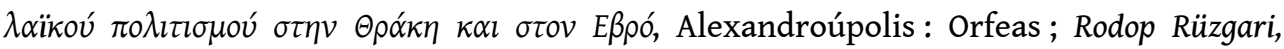
5 mai 2011, signale la présence lors d'une fête musulmane de Megalo Dereio d'élèves de l'école Isa Bey, Macédoine ex-Yougoslave (FYROM).

64. Brochure éditée par le dergâh d'Ali Seyyid Sultan, p. 2. Les données s'appuient sur l'hagiographie du saint.

65. Suraiya FAROQHI, 1976, p. 74-96; Mehmet Koç, 2011, Seyyid Ali Sultan Derghâhı ve Osmanl Eserleri, Istanbul: Dünden Bugüne. Ainsi, à la fin du $\mathrm{xIX}^{\mathrm{e}}$ siècle, le tekke constituait un complexe autonome au plan économique comptant 80 derviches, 2500 moutons, 200 bœufs, 5 moulins, 8 étables, 8 greniers, une fabrique d'huile et de

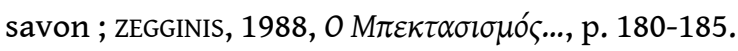

66. Entretiens avec le président du wakf du dergâh de Seyyid Sultan, mai 2011.

67. Le wakf désigne, en droit islamique, une donation en faveur d'une œuvre d'utilité publique et charitable. Par extension, la notion désigne le bien consacré. Le bien offert en usufruit devient alors inaliénable. Ce principe et ce type de propriété communautaire renvoient d'une part, à l'héritage ottoman dans la région; d'autre part, à la reconnaissance, en Thrace grecque, du droit islamique, dans certains secteurs concernant la minorité musulmane. TSITSELIKIs, 2006, The Shariatic Courts of Greek Thrace and the "Principle of Reciprocity" Regarding Minorities in Turkey and Greece;

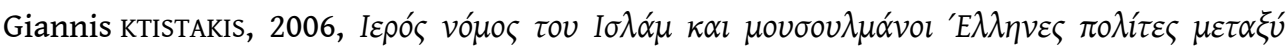

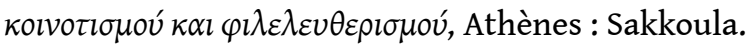

68. Brochure officielle du dergâh d'Ali Seyyid Sultan, p. 2-4.

69. Visite du tekke en mai 2011. Également Panagiotis KARAKATSANIS, 1992-1994, "To

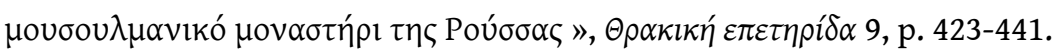

70. Entretiens en mai 2011 au village de Roussa. 


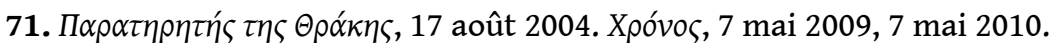

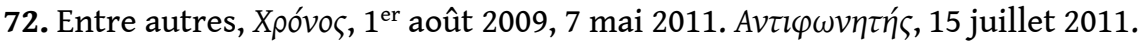

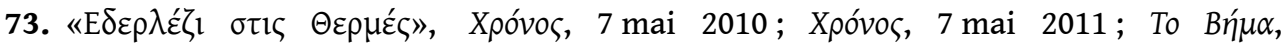
23 janvier 2011.

74. Entretiens juillet 2010, mai 2011.

75. Entretiens juillet 2010, mai 2011.

76. Entretiens juillet 2010, mai 2011.

77. Entretiens juillet 2010, mai 2011.

78. Entretiens juillet 2010, mai 2011.

79. Entretiens juillet 2010, mai 2011.

80. Katerina MARCOU, 2002, «Les Pomaques de Thrace grecque et leurs choix langagiers ", Études balkaniques 9, p. 41-51.

81. NEUBERGER, 2004, The Orient Within; Stoan RAJCEVSKI, 2004, The Mohammedan Bulgarians (Pomaks), Sofia: National Museum of Bulgarian Books and Polygraphy; Klaus STEINKE, Christian voss (eds.), 2007, The Pomaks in Greece and in Bulgaria, A Model Case for Borderland Minorities in the Balkans.

82. Alexandre POPOVIĆ, Gilles GRIVAUD (dir.), 2011, les Conversions à l'islam en Asie mineure et dans les Balkans aux époques seldjoukide et ottomane: bibliographie raisonnée (1800-2000), Athènes : École française d'Athènes; Tijana KRSTIC, 2011, Contested Conversions to Islam, Narratives of Religious Changes in the Early Modern Ottoman Empire, Stanford: Stanford University Press; Mercedes GARCIA-ARENAL (dir.), 2002, Conversions islamiques: identités religieuses en islam méditerranéen, Paris : Maisonneuve et Larose.

83. TSIBIRIDOU, les Pomaks..., chapitre 1, p. 29-79.

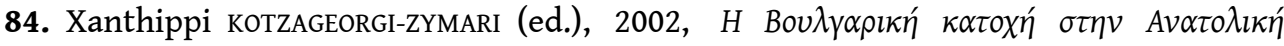

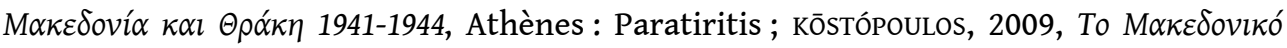
$\tau \eta \varsigma \Theta \rho \alpha ́ \kappa \eta \zeta$.

85. Notamment: Archives de l'État grec, archives I. Metaxás, dossier 36, du 5 mai 1936

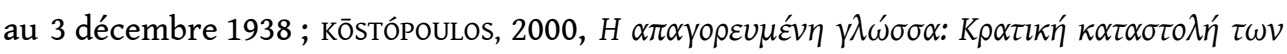

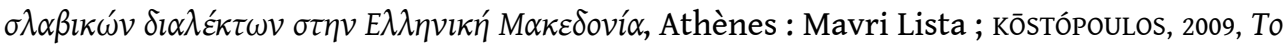

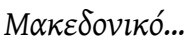

86. Entretiens à Xanthi et Komotini, juillet 2009, mai 2010.

87. MARKou, 2002, "Les Pomaques de Thrace grecque et leurs choix langagiers", p. 41-45.

88. Entretiens à Xanthi et Komotini, juillet 2009, mai 2010. Olga DIMITRIOU, 2004, "Prioritizing 'Ethnicities': The Uncertainty of Pomakness in the Urban Greek Rhodope”, Ethnic and Racial Studies 27-1, pp. 104-105; TSITSELIKIS, "Muslims in Greece" in POTZ R., WIESHAIDER W., 2004, Islam and the European Union, Louvain: Peeters, pp. 79-85.

89. Voir le journal gréco-pomaque Zagalisa.

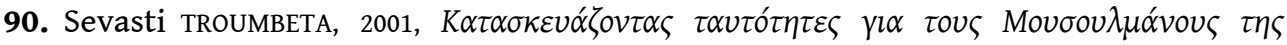

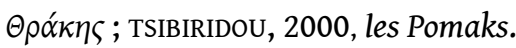

91. TRUMBETA, 2013, Physical Anthropology: Race and Eugenics 1880s-1970s, Leiden-Boston: Brill. 
92. «Agriens », 1774, le Grand vocabulaire françois, Paris, p. 486 ; Nikolaos XYROTIRIS, 1996,

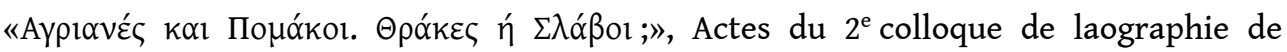
l'espace nord-helladique, Komotini, 19-22 mars 1995, Salonique: IMXA, p. 333-356;

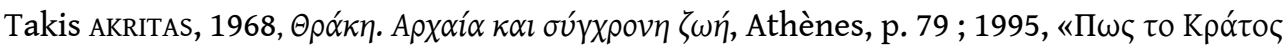

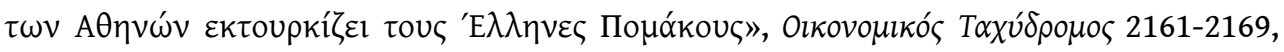
p. 107-109.

93. NEUBERGER, 2004, The Orient Within; Evangelos KARAGIANNIS, "An Introduction to the Pomak Issue in Bulgaria”, traduction anglaise de „Eine Einführung in die pomakische Frage“, in KARAGIANNIS, 1997, Zur Ethnizität der Pomaken Bulgariens, Münster: LIT, p. 34-52; STEINKE, vOsS, 2007, The Pomaks of Greece and Bulgaria: A Model Case for Borderland Minorities in the Balkans; LORY, 1987, "Une communauté musulmane oubliée: les Pomaks de Lovec", Turcica 19, p. 95-117; LORY, 1985, le Sort de l'héritage ottoman en Bulgarie: l'exemple des villes bulgares 1870-1900; Tsevtana GEORGIEVA, 2001, "Pomaks : Muslim Bulgarians", Islam and Christian-Muslim Relations 12-3, pp. 303-316; Nadège RAGARU, 2010, «Faire taire l'altérité : politique de la langue et mobilisations linguistiques au temps de l'assimilation forcée des Turcs de Bulgarie 1984-1989 ", Cultures et conflits 79-80, p. 73-96.

94. Kristen GHODSEE, 2009, Muslims Lives in Eastern Europe. Gender: Ethnicity and the Transformation of Islam in Post-Socialist Bulgaria, Princeton: Princeton University Press, pp. 37-38. Également, le site des Jeunes turcs de Thrace occidentale :

http://gencbatitrakyaturkleri.tr.gg/Pomaklar-Oz-ve-Oz-Turktur--k1-English-k2-.htm [consulté le 14 mars 2016].

95. Entretiens, mai 2011.

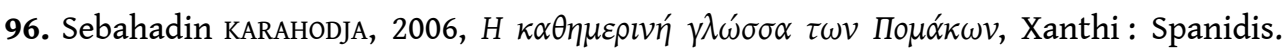
Voir les activités du Centre d'études pomaques (créé en 1997 à Komotini), de l'Association panhellénique des Pomaques (établie en 2009 à Komotini), de l'Association culturelle des Pomaques de la préfecture de Xanthi. Ces instances sont notamment financées par un homme d'affaire grec, Prodromos Emfietzoglu. Ces associations éditent le périodique Zagalisa, publication militante hellénophone et hellénophile visant la promotion d'une identité pomaque.

97. Xمóvos, 30 septembre 2010. Une interview du musulman Imam Ahmet, directeur du journal pomaco-hellénisant Zagalisa, semble avoir précipité le conflit. Entretiens juillet 2010, mai 2011.

98. Entretiens juillet 2010, mai 2011.

99. Zagalisa, 11 février 2011.

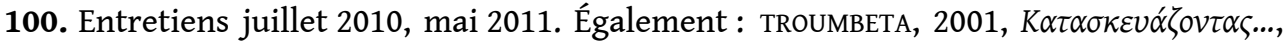
p. 77-158 ; Iannis FRANGOPOULOS, 1994, «La minorité musulmane et les Pomaques de la Thrace : entre islam et ethnisme », CEMOTI 17, p. 152-166.

101. Entre autres: Conseil de l'Europe, Charte européenne des langues régionales ou minoritaires, Strasbourg, 5 novembre 1992 : http://conventions.coe.int/treaty/fr/ Treaties/Html/148.htm [consulté le 14 mars 2016].

102. Clayer, BOUgAREL, 2013, les Musulmans..., p. 215-267.

103. Entretiens, juillet 2010, mai 2011. 
104. Pour les mobilisations alévies en Turquie et Allemagne, Élise MASSICARD, 2012, The Alevis in Turkey and Europe: Identity and Managing Territorial Diversity, Oxford: Routledge, 2012.

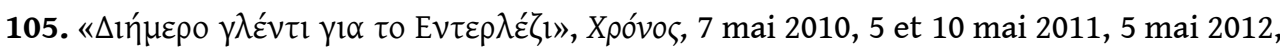

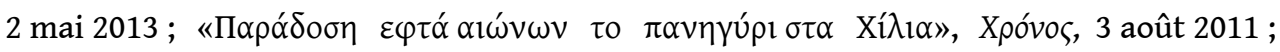
Olivier GIVRE, 2006, Un rituel "balkanique " ou un rituel dans les Balkans? Approche anthropologique $d u$ kourban en Bulgarie et en Grèce $d u$ Nord, thèse de doctorat en sociologie-anthropologie, Université de Lyon II, soutenue le 13 novembre 2006; Entretien avec la musicologue et folkloriste Miranda TERzopoulou, 2012, «Dans la

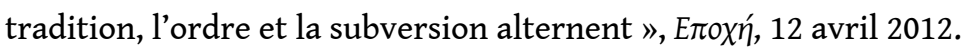

106. Büyük Dervent en turc, Goljam Dervent en bulgare.

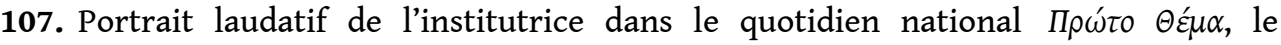

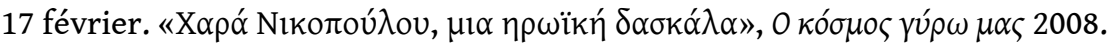

108. Le village, situé à $5 \mathrm{~km}$ de la frontière bulgare, s'inscrit dans une commune plus large d'environ 2100 habitants (2011) incluant en outre les villages de Roussa, Ieriko, Goniko, Mega Dereio, Petrolofos, Sidirochori.

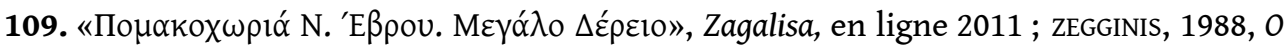

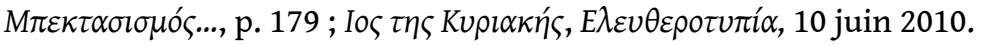

110. Selon l'universitaire bulgare Lyubomir мILETIсн, 1918, The Destruction of the Thracian Bulgarian in 1913, Bulgarian Academia of Science, pp. 290-302.

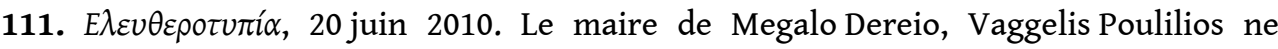

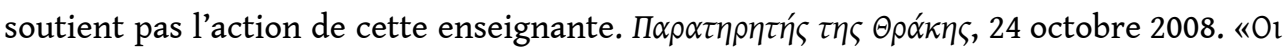

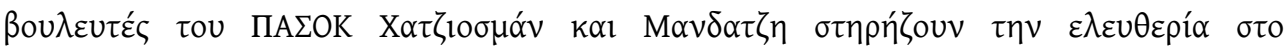

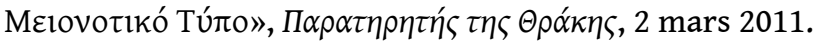

112. Question déposée par le député Ahmet Haciosman, au Parlement le

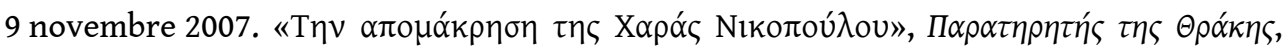
24 octobre 2008.

113. Xрóvoৎ, 8 février 2008. L'agresseur a été condamné à 10 mois de prison le

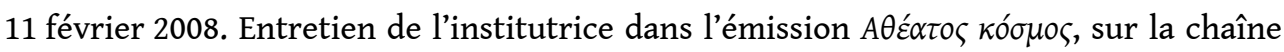

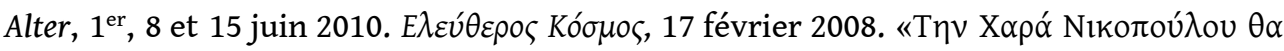

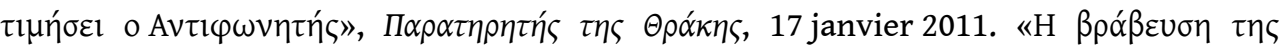

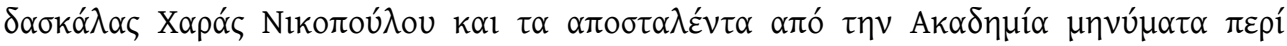

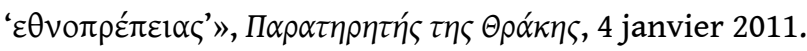

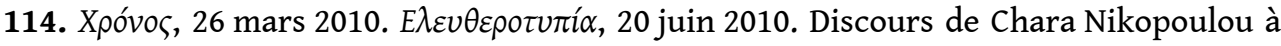
la préfecture de Salonique, 14 avril 2010, http://www.greekalert.com/2010/04/t.html [consulté le 14 mars 2016].

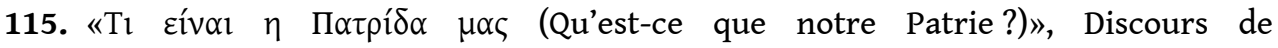
Chara Nikopoulou à l'occasion du prix que lui a décerné la préfecture de Salonique pour l'importance de son travail « éducatif et national », 10 avril 2010, https://www.youtube.com/watch?v=VfAGAr49Tw4 [consulté le 14 mars 2016].

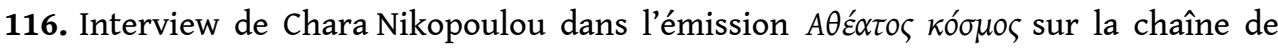
télévision grecque Alter, $1^{\mathrm{er}}$ juin 2010.

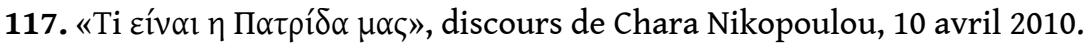




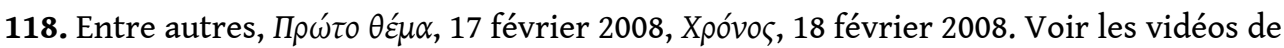
la cérémonie du 25 mars 2008, 2009, 2010 à Megalo Dereio. Articles correspondants sur

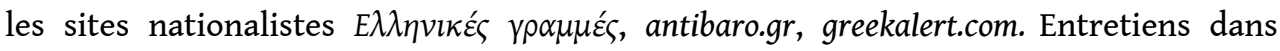

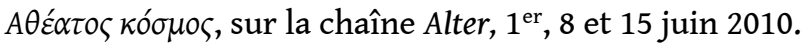

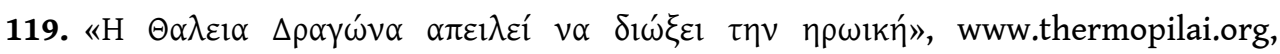
29 mars 2010.

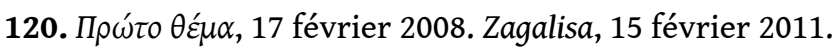

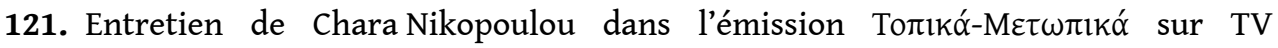
Salonique, le 12 février 2008.

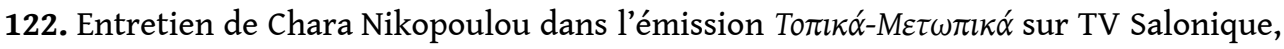
le 12 février 2008.

123. Entretiens à Komotini, Xanthi, Roussa, Mikro Dereio, mai 2011. Voir les journaux locaux turcophones Millet (Xanthi), Gündem, Birlik (Komotini). Également: «Büyük Derbent huzursuzluk devam ediyor ", Azınlınkça, 6 janvier 2008 ; « Hara Nikopoulou ile ilgili hepimizin düşunmesi gereken dersler », Azınlınkça, 29 juin 2010.

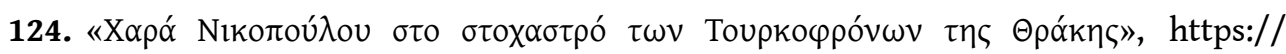
ellas2.wordpress.com/, 6 janvier 2010.

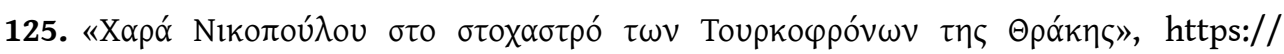

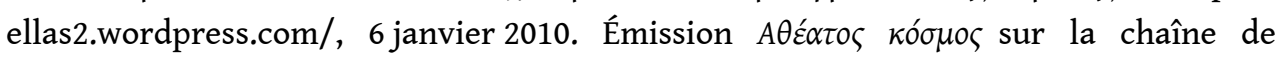
télévision grecque Alter, $1^{\mathrm{er}}$ juin 2010.

126. Éloges décernées par l'Académie d'Athènes, la préfecture de Salonique, des

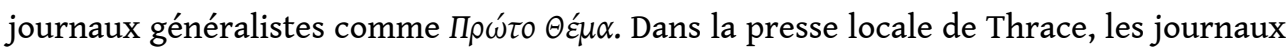

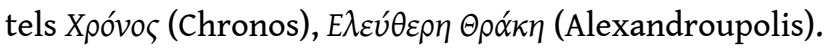

127. Zagalisa, 3 septembre 2009, 10 août 2009.

128. Millet, 16 décembre 2010.

129. Entretiens, juillet 2010, mai 2011.

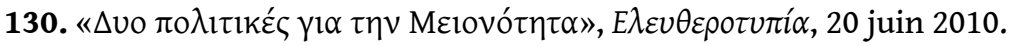

131. Rapport du Conseil consultatif de Thrace du 27 juillet 1964, cité par KōsTóPoulos, l'un des rares chercheurs à avoir eu accès à ce matériel sensible, Io $\tau \eta \varsigma K v \rho \imath \alpha \hbar n \zeta$,

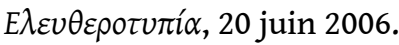

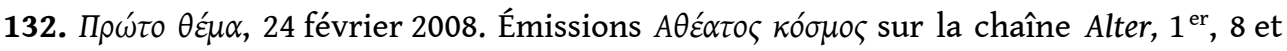

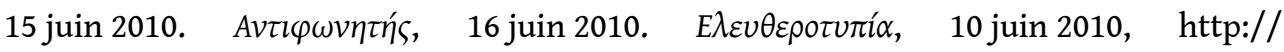
www.azinlikca.net/, 10 janvier 2010. www.bttadk.org, 16 juillet 2010. Xрóvos,

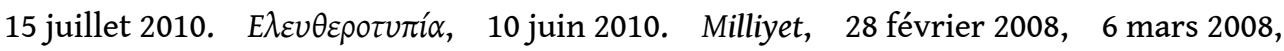
25 mai 2009, 18 mars 2010, 13 mai 2010, 9 décembre 2010. Gündem, 14 mars 2008, 7 mai 2010.

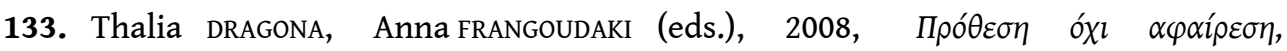

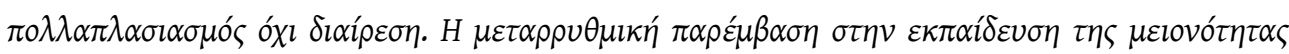

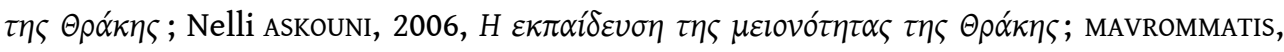

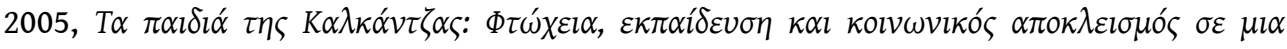

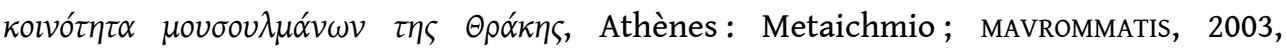
"Constructing Identities for the Thracian Muslim Youth: the Role of Education", p. 113-123. 


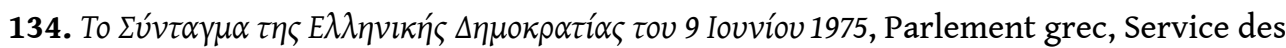
études, 1992, article 16.

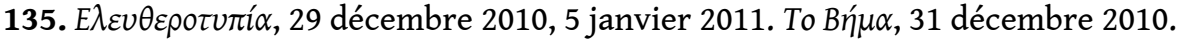

136. Discours du métropolite de Kalavryta et Aigieleias, 18 avril 2010, site du métropolite de Kalavryta, http://www.im-ka.gr/.

137. Voir les sites http://www.antibaro.gr/, http://greekalert.gr/, http:// www.antinews.gr/, ellpalmos.blogspot. Xрóvos, 27 mars 2008, 5 juin 2010, 13 janvier 2011.

138. TSITSELIKIS, Old and New..., p. 97-117.

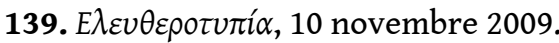

140. Thalia DRAGONA, Anna FRANGOUdAKI (eds.), 1997, $T i^{\prime} v \alpha l \eta \Pi \alpha \tau \rho i ́ \delta \alpha \mu \alpha \varsigma$, Athènes : Alexandria.

141. DRAGONA, FRANGOUDAKI (eds.), 2008, Прó $\theta \varepsilon \sigma \eta . . .$, site du ministère de l'Éducation

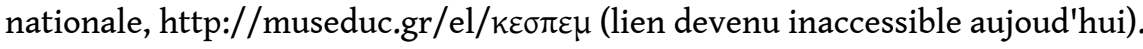

142. Voir la thèse non publiée de Vemund AARBAKKE, 2000, The Muslim Minority of Thrace, Université de Bergen, qui exploite principalement la presse turcophone locale.

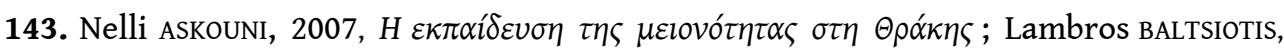

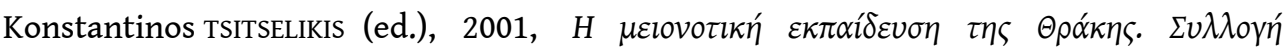

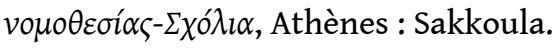

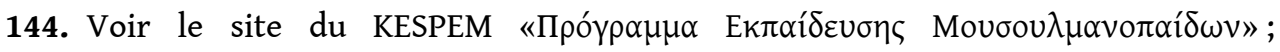

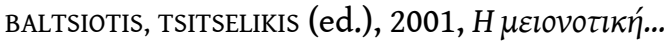

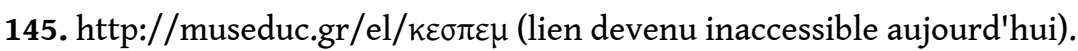

146. Ici, le terme Orientaliste désigne un spécialiste de langues, cultures et civilisations dites "orientales ». Voir ici les travaux très intéressants développés dans l'ouvrage posthume de l'archéologue et historien britannique Frederick HASLUCK, 1929, Christianity and Islam under the Sultans; Gilles VEINSTEIN (dir.), 2005, Syncrétismes et hérésies dans l'Orient seldjoukide et ottoman (XIV ${ }^{e}-\mathrm{XVIII}{ }^{e}$ siècles).

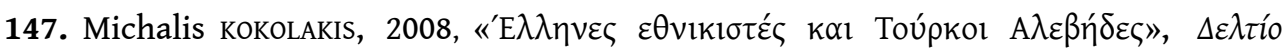

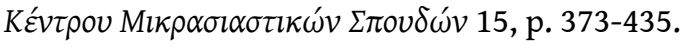

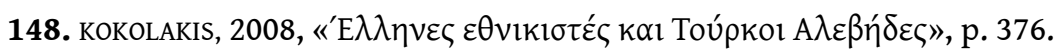

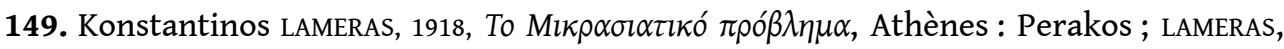

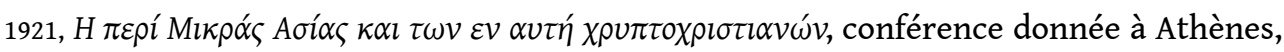
Athènes : Kallergis.

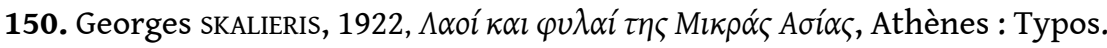

151. Les travaux de HASLUCK développent la problématique des influences ou des survivances chrétiennes au sein de l'islam. Pour cet auteur, les proximités entre l'islam des derviches et d'autre part le christianisme reflèteraient un processus socio-culturel de long terme, le passage graduel du christianisme à l'islam dans certaines régions ottomanes.

152. Discours du 3 février 1920 d'Eleftherios Vénizélos. Nikos PETSALIS-DIOMIDIS, 1978, Greece at the Peace Conference (1919), Salonique : Institut d'études balkaniques, p. 348-349.

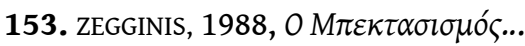


154. Miranda TERzopoulou, 2009, communication "The Evolution of a Bektashi Panayir in Greek Thrace/Kimlik", Conférence internationale consacrée à Haci Bektash Veli, Ankara, 19-21 octobre.

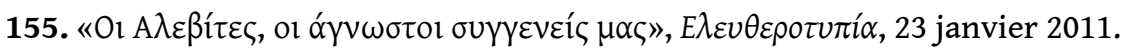

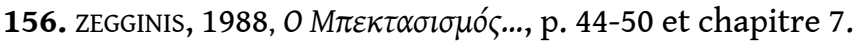

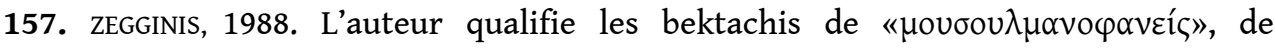
personnes «d'apparence musulmane», p. 247; Manolis VARVounIS, 1993, "A Contribution to the Study of Influences of Christian upon Moslem Customs in Popular Worship", Journal of Oriental and African Studies 5, pp. 75-89; vaRvounIs, 1995, "Christian Orthodox and Muslim Popular Religious Customs : a Study of Influences and Practices",

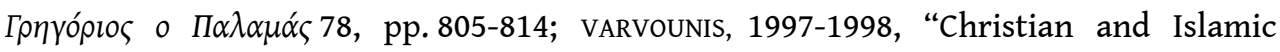
Parallel Cultural Traditions in the Popular Culture of the Balkan People", Journal of

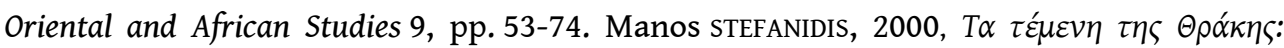

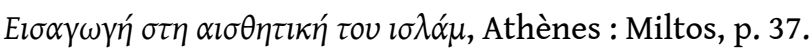

158. La notion de "région intermédiaire " est un concept clé dans la vision géopolitique de Dimitris Kitsikis.

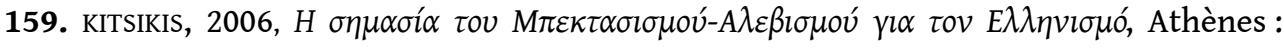
Ekati, p. 9, 20, 35.

160. Thierry ZARCONE, 2009, le Soufisme : voie mystique de l'islam.

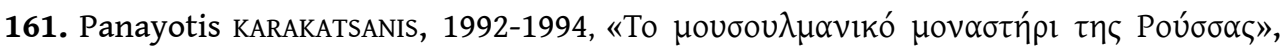

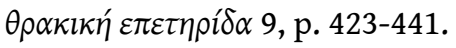

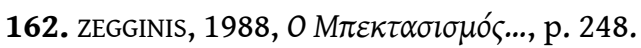

163. Le bogomilisme fut condamné par l'Église catholique romaine, les empereurs byzantins et des princes serbes. Dmitri OBOLENSKY, 1948, The Bogomils, Cambridge: Cambridge University Press; Richard CRAMPTON, 1997, A Concise History of Bulgaria, Cambridge: Cambridge University Press; Nina GARSOIAN, 1967, The Paulician Heresy, La Haye: Mouton; Joseph Hoffman, 1983, "The Paulician Heresy: A Reappraisal", Patristic and Byzantine Review 2-2, pp. 251-263.

164. « Nouveau drame de l'immigration en Grèce », Le Monde, 15 novembre 2013.

165. En Turquie, entre $15 \%$ et $20 \%$ de la population totale serait alévie au début du $\mathrm{xxI}^{\mathrm{e}}$ siècle. Pour une étude des mouvements identitaires alévistes en Turquie et dans la diaspora européenne, au tournant des $\mathrm{xx}^{\mathrm{e}}$ et $\mathrm{xXI}^{\mathrm{e}}$ siècles: MASSICARD, 2005, l'Autre Turquie : le mouvement aléviste et ses territoires; Markus DRESSLER, 2011, "Making Religion through Religious Secularist Discourse: The Case of Turkish Alevism", in Markus DRESSLER, Arvind MANDAIR (eds.), Secularism and Religion Making, Oxford: Oxford University Press, pp. 188-208.

166. À partir du début 2008, l'enseignante multiplie les entretiens, déclarations et discours dans les médias. Nikopoulou participe activement à la fondation, en 2012, du

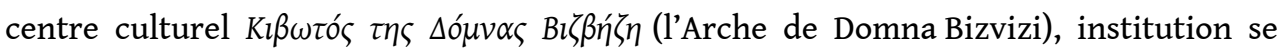
proposant «d'étudier et de promouvoir l'esprit helléno-orthodoxe, l'approche universitaire du penser grec, des valeurs diachroniques et et oecuméniques de

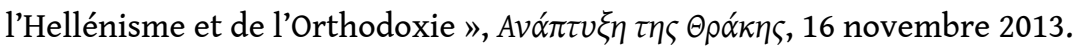

167. To Bท́ $\mu \alpha, 3$ janvier 2010. Plusieurs cadres du parti d'extrême droite LAOS assistent aux cérémonies du 25 mars 2010 à Megalo Dereio. 
168. La brochure présentant le tekke de Roussa inscrit clairement le lieu sacré dans la culture turque, valorisant, par ailleurs, l'héritage kémaliste. Xpóvoৎ, 28 juin 2008.

\section{RÉSUMÉS}

En Europe du Sud-Est, l'alévisme et le bektachisme représentent deux courants étroitement liés, dont les racines plongent dans l'histoire ottomane de la région. Inscrit dans la tradition musulmane soufie, reprenant des éléments du chiisme, l'alévisme combine des influences variées, parmi lesquelles le chamanisme. En Grèce, ce courant religieux est représenté en Thrace, dans un espace culturel gréco-turco-bulgare. Quelques communautés résident en milieu montagneux et limitrophe, au nord des départements de Xanthi et de l'Évros. Cultivant une mémoire communautaire, les alévis de Thrace semblent aujourd'hui tiraillés entre repli, assimilation au sunnisme, sécularisation et reconstitution de réseaux transfrontaliers. Peu connus en Grèce, les alévis ont souvent été envisagés sous le prisme du christianisme par la littérature hellénophone en sciences sociales, suivant en cela les thèses d'orientalistes européens des $\mathrm{XIX}-\mathrm{XX}$ siècles. Longtemps ignorés ou méprisés en tant que communauté spécifique, les bektachis de Thrace sont au contact du nationalisme turc - promu par des acteurs locaux ou internationaux - mais aussi de réseaux alévis transétatiques récemment revitalisés. Une confrontation scolaire survenue au début $\mathrm{du} \mathrm{xxI}^{\mathrm{e}}$ siècle dans un village alévi et largement pomaque permet d'observer ces jeux entre identité et altérité religieuse, discours nationaux et instrumentalisations. Cette étude de cas - où s'articulent logiques locales, nationales et politique internationale - rappelle que l'islam demeure, en Grèce, une question sensible.

In South-Eastern Europe, Alevism and Bektashism constitute two tightly connected religious movements whose roots go down to the Ottoman history of this area. This branch of Islam belongs to Sufi traditions, integrates elements of Shiism as well as various influences, among which shamanism. In Greece, alevism-bektashism still exists in Western Thrace, in a TurkishBulgarian-Greek cultural environment. A few alevi communities live especially in mountainous villages, in the North of the Xanthi and Evros administrative circonscriptions. Cultivating a community memory, the Alevis of Western Thrace seem to be torn today between withdrawal, assimilation to sunnism, secularization and revitalization of cross-border networks. As a minority within the "Muslim minority" of Thrace, this religious group is little known in Greece. Greek social sciences scholars who followed, in this respect, the readings of prominent 19th-20th centuries European Orientalists have often studied this group under the prism of christianism. Long ignored or despised as a specific community, the Western Thrace bektashis are also directly in contact with Turkish nationalism - promoted by local or international protagonists - and with newly revitalized Alevi networks. A school clash in an alevi - and predominantly Pomak - village enables us to examine the interplay of religious identity and otherness, national narratives and political strategies. This case study - in which local, national and foreign policy factors are intertwined - reminds us that Islam has remained a highly sensitive issue in Greece.

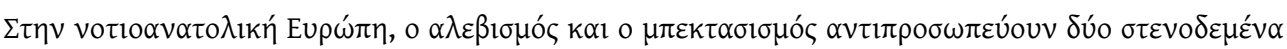

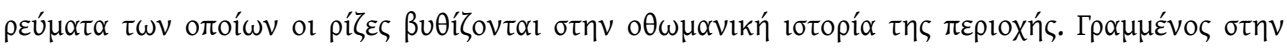

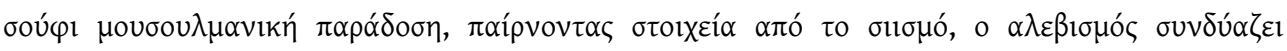

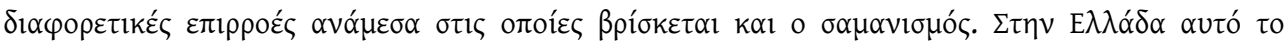




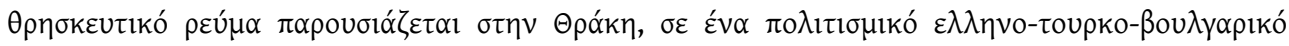

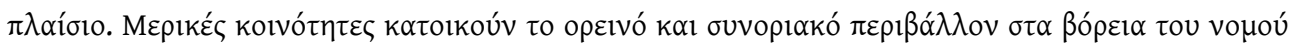

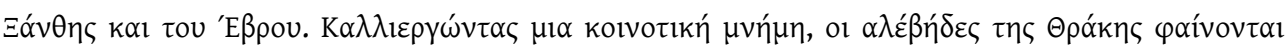

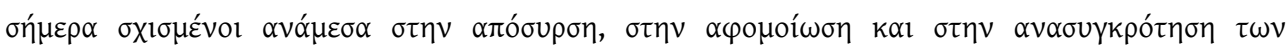

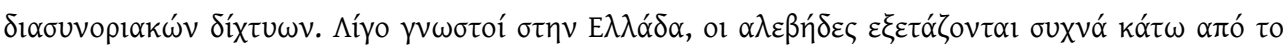

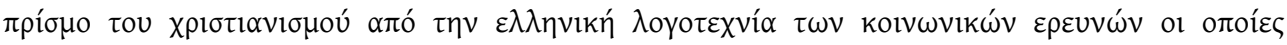

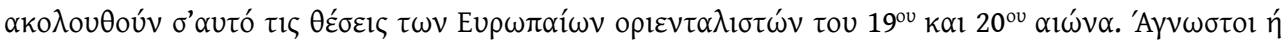

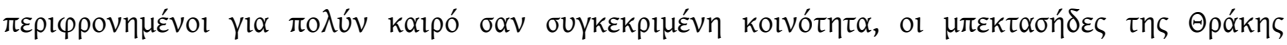

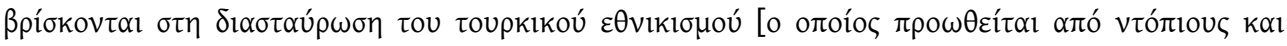

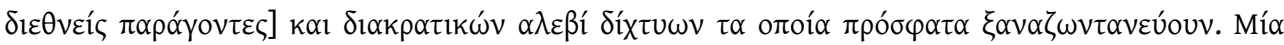

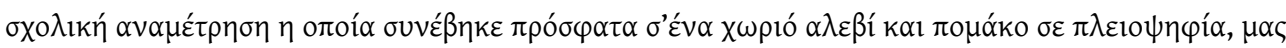

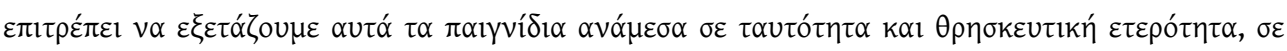

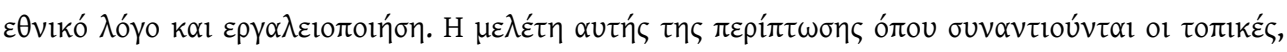

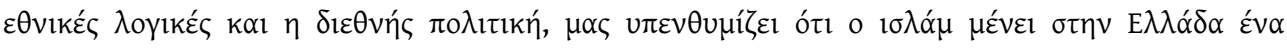

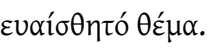

\section{INDEX}

Index géographique : Grèce, Thrace

Thèmes : Religion et politique

motsclestr Heterodoks İslam, Alevilik, Bektaşilik, Trakya, Yunanistan, Yirmi birinci yüzyıl, Din ve siyaset

Index chronologique : vingt-et-unième siècle

Keywords : Heterodox Islam, Alevism, Bektachism, Thrace, Greece, Twenty first century, Religion and Politics

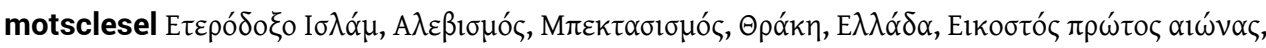

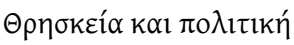

Mots-clés : islam hétérodoxe, alevisme, bektachisme

motsclesmk ИНОСЛАВНИТЕ ИСЛАМОТ, АЛЕВИЗМОТ, БЕКТАШИСТВОТО, ТРАКИЈА, ГРЦИЈА, ДВАЕСЕТ И ПРВИОТ ВЕК, РЕЛИГИЈАТА И ПОЛИТИКАТА

\section{AUTEUR}

\section{ISABELLE DÉPRET}

CIERL, Université Libre de Bruxelles 